\title{
Geminography: the crystallography of twins
}

\author{
Hans Grimmer*,I and Massimo Nespolo*,II

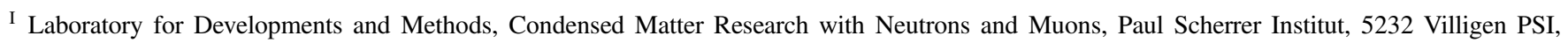 \\ Switzerland \\ II Université Henri Poincaré Nancy 1, Laboratoire de Cristallographie et de Modélisation des Matériaux Minéraux et Biologiques (LCM3B), \\ UMR - CNRS 7036, Faculté des Sciences et Techniques, Boulevard des Aiguillettes, BP 239, F-54506 Vandœuvre-lès-Nancy cedex, France
}

Received November 26, 2004; accepted February 7, 2005

Twinning / Coincidence-site lattice / Diffraction from twins / Merohedry / Geminography

\begin{abstract}
The geometric theory of twinning was developed almost a century ago. Despite its age, it still represents the fundamental approach to the analysis and interpretation of twinned crystals, in both the direct and the reciprocal space. In recent years, this theory has been extended not only in its formalism (group-subgroup analysis, chromatic symmetry) but also in its classification of special cases that were not recognized before. The geometrical theory of twinning is thus reviewed here with emphasis on lattice aspects and recent developments. The classification of various types of twins starts with Friedel's well-known scheme, which distinguishes four cases according to whether the twin index $n$ is equal to or larger than 1 and whether the obliquity $\omega$ is equal to or larger than 0: the computation of these quantities is discussed in detail. It is shown that the concept of obliquity is not sufficient to characterize the pseudo-symmetry of a lattice, and the consequent twinning, in the case of manifold twins. The application of the theory of coincidence-site lattices to twinning is presented. Finally, the effect of twinning on the diffraction pattern is illustrated with a number of examples.
\end{abstract}

\section{Introduction}

\subsection{Scope of the article}

"Geminography" is the term introduced by J. D. H. Donnay (Takeda, 1975; Nespolo and Ferraris, 2003) to indicate the branch of crystallography specifically dealing with twinned crystals. Geminography dates back to the origin of crystallography, and has found its most satisfying expression in the reticular theory developed by the socalled "French school", brought to maturity by Friedel (1904, 1926). Several reviews of geminographical problems exist, the most recent being in Hahn and Klapper

\footnotetext{
* Correspondence authors (e-mail: hans.grimmer@psi.ch and massimo.nespolo@lcm3b.uhp-nancy.fr)
}

(2003). The purpose of this article is to present an operative review of some aspects that are not treated elsewhere (modern extensions of the classical geminography theory, applications of the Coincidence-Site Lattice theory, interpretation of diffraction patterns of twins), as well as to summarize the practical approach of deriving and computing fundamental geminographical variables (e.g. twin lattice cell, twin index, twin obliquity).

Let us consider oriented associations of crystals in which each crystal (the "individual") has the same (or nearly the same) chemical composition and crystal structure (homophase oriented associations). A homophase oriented association of two or more macroscopic, congruent or enantiomorphic, individuals is called a twin if its occurrence is not unique and if the orientation relations between the individuals are 'crystallographic', namely they can be expressed either by an inversion, a mirror reflection, a 2fold rotation or (within some tolerance) a 3-, 4- or 6-fold rotation. The mirror plane must be parallel to a lattice plane or perpendicular to a lattice row, the rotation axis parallel to a lattice row or perpendicular to a lattice plane.

The individuals are homogeneous and as such they are described by an ordinary three-dimensional triperiodic space group. The edifice formed by their association is instead heterogeneous: it can be described by a special kind of point group (polychromatic twin point group), but not by a space group.

\subsection{Definitions}

To describe the orientation relations between pairs of individuals in a twin we need to review a few concepts (see Hahn and Klapper, 2003; Nespolo, 2004).

Isometry denotes a mapping of three-dimensional space onto itself that leaves all distances and angles invariant.

Symmetry operation of an individual is an isometry that maps the individual onto itself.

Twin operation is the linear part of an isometry that maps one of the individuals of the pair under consideration onto the other, i.e. we consider only the relative orientation of the two individuals, neglecting a possible translation part of the isometry. 
Twin element is the geometric element (mirror plane, inversion centre, rotation or rotoinversion axis) about which the twin operation is performed together with the indication of its type $(2,3,4,6, \overline{3}, \overline{4}$, or $\overline{6})$ in case of an axis.

Composite symmetry. The point groups $\mathscr{H}_{1}$ and $\mathscr{H}_{2}$ of both individuals belong to the same type $\mathscr{H}$. However, the orientation of the symmetry elements may be different for the two individuals. Let $\mathscr{H}^{*}$ consist of the symmetry elements that are common to the point groups $\mathscr{H}_{1}$ and $\mathscr{H}_{2}$ of the two individuals: $\mathscr{H}^{*}$ is thus the intersection group of $\mathscr{H}_{1}$ and $\mathscr{H}_{2}$. The composite symmetry $\mathscr{K}^{*}$ is the point group generated by $\mathscr{H}^{*}$ and the twin operation $k ; \mathscr{K}^{*}$ is a supergroup of $\mathscr{H}^{*}$. In the special case, where $\mathscr{H}^{*}=\mathscr{H}_{1}$ $=\mathscr{H}_{2}$, the composite symmetry $\mathscr{K}^{*}$ will be abbreviated to $\mathscr{K}$. We shall limit our attention to cases where $\mathscr{K}^{*}$ is a crystallographic point group. The number of elements in $\mathscr{K}^{*}$ is a multiple of the number of elements in $\mathscr{H}^{*}$. If $\mathscr{K}^{*}$ has $m$ times as many elements as $\mathscr{H}^{*}$ then the twin operation $k$ will generate $m$ different orientation states, which we may mark by different colours. In order to stress the fact that $\mathscr{K}^{*}$ characterizes the point group symmetry of a twin, not of an individual, the concept of (poly) chromatic twin point groups has been introduced (Curien and Le Corre, 1958; Nespolo, 2004).

Twin law is the set of all equivalent twin operations that transform one of the individuals of the pair under consideration onto the other. As we shall see below, the twin operations corresponding to the same twin law belong to the same left coset of $\mathscr{H}^{*}$ in $\mathscr{K}^{*}$.

Twin degree is the number of independent twin elements: first-degree twins and higher-degree twins are twins in which only one or more than one independent twin element exists, respectively.

Twin multiplicity is the number of different orientation states generated by the twin elements.

Order of a (finite) group is the number of group elements in that group.

Order of a group element $y$ and order of the twin element associated to $\mathscr{y}$ is the least positive integer $n$ for which the power $\mathscr{y}^{n}$ of $\mathscr{y}$ is equal to the identity.

Twofold twins are twins in which the individuals are related only by twin elements of order 2. First-degree twofold twins are called binary twins.

Manifold twins are twins in which at least one twin element has order higher than 2 .

\subsection{Coset analysis and dichromatic point group notation for binary twins}

For binary twins, the number of elements in $\mathscr{K}^{*}$ is twice as large as the number of elements in $\mathscr{H}^{*}$. The set of elements obtained by performing first an operation in $\mathscr{H}^{*}$, then the twin operation $k$ is denoted by $k \mathscr{H}^{*}$ and is called a (left) coset of $\mathscr{H}^{*}$ in $\mathscr{K}^{*}$. Each element of $\mathscr{K}^{*}$ lies either in $\mathscr{H}^{*}$ or in the coset $k \mathscr{H}^{*}$ :

$$
\mathscr{K}^{*}=\mathscr{H}^{*} \cup k \mathscr{H}^{*} \text {. }
$$

The coset $k \mathscr{H}^{*}$ defines the twin law that corresponds to $k$. Imagine one orientation state of a binary twin to be black and the other to be white. The elements of $\mathscr{H}^{*}$ map the black orientation state onto itself, similarly for the white orientation state; the elements of $k \mathscr{H}^{*}$ interchange the black and white orientation state. The elements of the coset will be marked by a prime, similarly as in the usual notation describing the symmetry of magnetically ordered crystals.

The Hermann-Mauguin symbol of point groups consists of symbols of symmetry operations generating the point group. In dichromatic notation for $\mathscr{K}^{*}$, the generating group elements belonging to $k \mathscr{H}^{*}$ are primed, those belonging to $\mathscr{H}^{*}$ are written without prime, as usual.

\section{Twinning by merohedry}

Let us start with the simplest case, merohedric twinning of merohedral crystals. A crystal is called merohedral if its point group $\mathscr{H}$ has less elements than the holohedry, i.e. the point group of the crystal lattice $\mathscr{D}$. In this chapter we consider the usual case of syngonic merohedry, where the lattice has no specialized metric increasing its symmetry. The individuals of a merohedric twin are then related by group elements of the holohedry that do not belong to the point group of the crystal. The possible cases are listed in Table 1.

Column $m$ gives the subgroup index of $\mathscr{H}$ with respect to $\mathscr{D}$. One speaks of hemihedry if $m=2$, of tetartohedry if $m=4$, of ogdohedry if $m=8$. There is one possible twofold merohedric twin law for hemihedral crystals, three for tetartohedral crystals, and seven for ogdohedral crystals. The last column gives for each of these cases a twofold twin operation, i.e. an operation that maps two of the individuals of the twin onto each other. The dots indicate the orientation of the corresponding symmetry elements (cf. Chapter 10 of Hahn, 2002). The twin can be described as an inversion twin in the cases marked $\overline{1}$; the twin operation then belongs to the Laue class of the crystal. Such twins have been called class I twins by Catti and Ferraris, 1976. If the merohedral crystal has a centrosymmetric point group (written in bold) then $\overline{1}$ cannot appear as twin operation because it is a symmetry operation of the crystal.

\subsection{Quartz examples}

$\alpha$-quartz has space group $P 3_{1} 21$ or $P 3_{2} 21$ for left- or right-handed crystals, respectively. Its lattice is therefore of Bravais type $h P$ and its point group is 321 . Table 1 shows that three merohedric twin laws are possible. The respective twins are known as Brazil twins, Dauphiné (or Swiss) twins, and combined Brazil-Dauphiné twins, also known as Leydolt or Liebisch twins.

The twin operation of a Brazil twin is normally described as reflection in a mirror plane $\{11 \overline{2} 0\}$. An alternative but equivalent description is as inversion twin. It makes the connection between the three twin laws more obvious: Dauphiné twins are in fact rotation twins with axis $2_{[001]}$, and the combined twins are reflection twins 
Table 1. Possible twin operations for twins by merohedry (adapted from Koch, 2004).

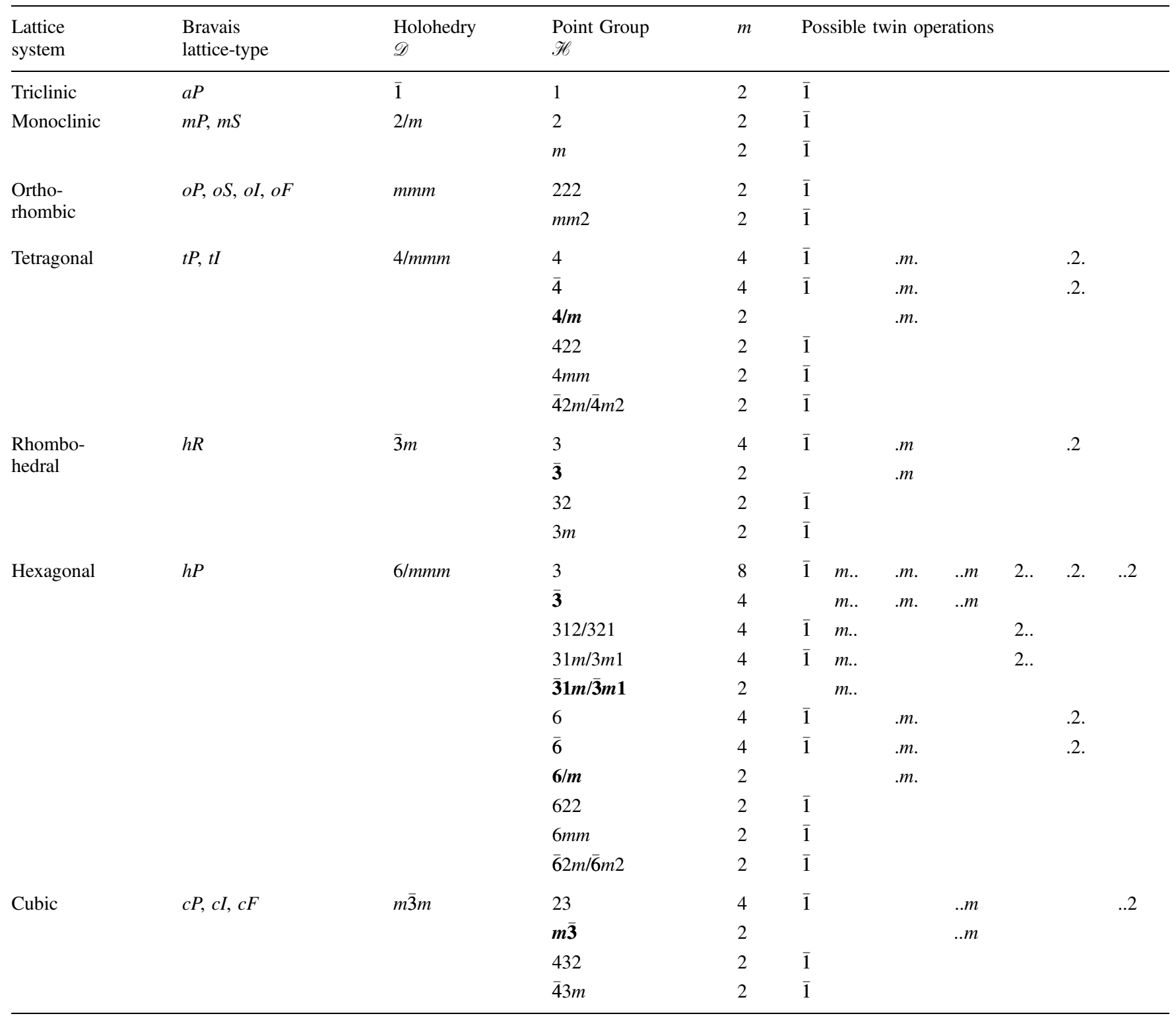

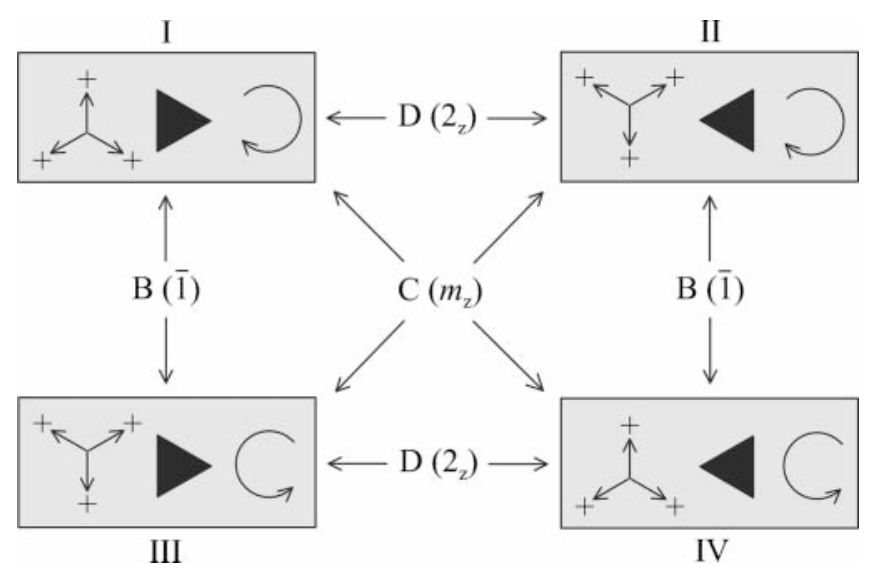

Fig. 1. The four fields (I-IV) show possible combinations of three properties in different orientation states of $\alpha$-quartz: orientation of the three electrical polar axes (triangle of arrows), orientation of etch pits on (0001) (solid triangle) and sense of the optical rotation (circular arrow). The arrows between the four fields show the possible combinations of two individuals in twins related by the Brazil law (B), the Dauphiné law (D) and the combined law (C) (reproduced from HahnKlapper (2003) with the permission of the IUCr). with mirror plane $m_{z}=m_{(001)}$, resulting from the combination of $\overline{1}$ and $2_{[001]}$ (see Fig. 1).

All the twin operations listed in Table 1 correspond to the special case where $\mathscr{H}^{*}=\mathscr{H}_{1}=\mathscr{H}_{2}$ and, therefore, $\mathscr{K}^{*}=\mathscr{K}$. The black-white point groups of merohedric twins in $\alpha$-quartz are given in Table 2.

The individuals of merohedric twins have all their symmetry axes parallel. But $\alpha$-quartz also has twins with inclined axes, e.g. Japan twins (also known as "La Gardette" twins), which will be discussed later as examples of twins by reticular pseudo-merohedry.

Table 2. Black-white notation for merohedric twins in $\alpha$-quartz.

$\alpha$-quartz: Point group $\mathscr{H}=321$

\begin{tabular}{ll}
\hline Twin law & $\mathscr{K}$ in black-white notation \\
Brazil & $\overline{3}^{\prime} \frac{2}{m^{\prime}} 1$ \\
Combined & $\overline{6}^{\prime} 2 m^{\prime}$ \\
Dauphiné & $6^{\prime} 22^{\prime}$
\end{tabular}




\section{The twin lattice}

The definition of the twin lattice has a more complex history than one might imagine. Friedel used the concept of a lattice, multiple of that of the individual, extending to the whole twinned edifice (Friedel, 1926, p. 425), but he did not explicitly introduce a definition of twin lattice. It was Donnay (1940) who defined the twin lattice as the lattice having for its primitive translations the edges of a cell, simple or multiple, endowed, either rigorously or approximately, with more symmetry than the crystal. This definition actually contains a common mistake. Let $\mathbf{L}_{\mathrm{T}}$ be the twin lattice, and $\mathbf{L}_{j}$ the lattice of the $j$-th individual $(j=1$, $2, \ldots N)$. By definition, the various $\mathbf{L}_{j}$ have point symmetries of the same type but different orientations. Only the symmetry elements that are common to all the $\mathbf{L}_{j} s$ are retained as symmetry elements of $\mathbf{L}_{\mathrm{T}}$, the others being lost. These together with the twin elements generate the symmetry of $\mathbf{L}_{\mathrm{T}}$. It follows that the symmetry of $\mathbf{L}_{\mathrm{T}}$ can be higher, equal, or lower than that of $\mathbf{L}_{j}$ (Buerger, 1954). The column CSL in Table 10 below gives in its rows with $c / a \neq 1$ examples where the the $\mathbf{L}_{j}$ have tetragonal symmetry and $\mathbf{L}_{\mathrm{T}}$ has higher (Bravais types: $c P, c l$ ), equal ( $t P$, $t I)$, lower $(o P, o C, o I, o F)$ or different $(h P)$ symmetry.

Assume that the lattices $\mathbf{L}_{j}$ have point groups $\mathscr{D}\left(\mathbf{L}_{j}\right)$ of type $\mathscr{D}$ and let $\mathscr{D}^{*}$ be the intersection group $\mathscr{D}^{*}=\mathscr{D}\left(\mathbf{L}_{1}\right)$ $\cap \mathscr{D}\left(\mathbf{L}_{2}\right) \cap \ldots \cap \mathscr{D}\left(\mathbf{L}_{N}\right)$, then $\mathscr{D}\left(\mathbf{L}_{\mathrm{T}}\right)$, the point group of $\mathbf{L}_{\mathrm{T}}$, is a supergroup of $\mathscr{D}^{*}$, which may not contain a subgroup of type $\mathscr{D}$.

The twin elements are symmetry elements for $\mathbf{L}_{\mathrm{T}}$. A twin element cannot be a symmetry element for the individual; otherwise the individuals would have orientations that are equivalent, giving rise to a parallel growth, not a twin. A twin element is thus a symmetry element for the twin lattice but not for the individual.

By a reflection (rotation) twin in its broad sense we shall understand a twin where the lattices $\mathbf{L}_{1}$ and $\mathbf{L}_{2}$ of two of the individuals are related by a mirror reflection in a lattice plane with low indices $(h k l)$ (a crystallographic rotation about a lattice row with low indices $[u v w])$. Notice that this does not imply, except for holohedral crystals, that the two individuals are related by such operations.

Consider first a reflection twin with twin plane $(h k l)$. All vectors in this plane are left invariant by the mirror reflection. We conclude: The vectors common to the lattices $\mathbf{L}_{1}$ and $\mathbf{L}_{2}$ of the two individuals of a reflection twin form at least a 2-dimensional lattice.

Assume there exists a lattice row $[u v w] \perp(h k l)$. The vector $[u v w]$ of $\mathbf{L}_{1}$ is mapped onto - $[u v w]$, which belongs not only to $\mathbf{L}_{2}$ but also to $\mathbf{L}_{1}$. It follows that the vectors common to $\mathbf{L}_{1}$ and $\mathbf{L}_{2}$ form a 3-dimensional lattice, called the twin lattice $\mathbf{L}_{\mathrm{T}}$.

Consider a rotation twin with twin axis $[u v w]$. All vectors in this direction are left invariant by the rotation. We conclude: The vectors common to the lattices of the two individuals of a rotation twin form at least a 1-dimensional lattice.

Consider now the special case of a 2 -fold rotation and assume that there exists a lattice plane $(h k l) \perp[u v w]$. The 2-fold rotation about $[u v w]$ maps each vector $[r s t]$ of $\mathbf{L}_{1}$ in $(h k l)$ onto [rst], which belongs not only to $\mathbf{L}_{2}$ but also to $\mathbf{L}_{1}$. It follows that the vectors common to $\mathbf{L}_{1}$ and $\mathbf{L}_{2}$ form a 3-dimensional lattice, called the twin lattice $\mathbf{L}_{\mathrm{T}}$.

For a reflection twin with twin plane $(h k l)$ and for a rotation twin with 2 -fold twin axis [uvw] the existence of a pair $(h k l) \perp[u v w]$ is necessary and sufficient for the vectors common to $\mathbf{L}_{1}$ and $\mathbf{L}_{2}$ to form a 3-dimensional lattice. It is called the twin lattice $\mathbf{L}_{\mathrm{T}}$. The ratio of the volumes $V_{\mathrm{T}}$ and $V$ of primitive cells for $\mathbf{L}_{\mathrm{T}}$ and $\mathbf{L}_{1}$, respectively, $n=V_{\mathrm{T}} / V$, is called twin index (or multiplicity).

The search for possible twin elements therefore starts with the search for directions and planes that are mutually perpendicular. In the case of cubic crystals, a direction and a plane with the same indices are always perpendicular. In the case of crystals with a 3-, 4- or 6-fold axis, there is a lattice plane perpendicular to a lattice direction for every value of the axial ratio $c / a$ if the lattice direction is either parallel or perpendicular to the principal symmetry axis. For orthorhombic and monoclinic crystals there is a lattice plane perpendicular to a lattice direction for all values of the lattice parameters if the lattice direction is parallel to a symmetry axis. Finally, for triclinic crystals there is in general no pair of mutually orthogonal lattice plane and lattice direction. For special values of the lattice parameters there are additional mutually orthogonal pairs, as shown by Fortes (1977).

Nevertheless, it is not infrequent to find twofold twins where the twin axis (or plane) is not exactly perpendicular to a lattice plane (or direction). In these cases the twin elements are pseudo-symmetry elements for the twin lattice, which will be discussed in Section 3.3. When the twin plane and the twin axis of a twofold twin are perpendicular, one speaks of twinning by Twin Lattice Symmetry (TLS). When they are not exactly perpendicular, one speaks of twinning by Twin Lattice Quasi Symmetry (TLQS) (Donnay and Donnay, 1974). A finer classification is described below in terms of twin obliquity and twin index.

The situation is more complicated in the case of manifold twins, because in this case the existence of a lattice plane $(h k l)$ perpendicular to the twin axis $[u v w]$ is neither a necessary nor a sufficient condition for the two lattices to have three non-coplanar vectors in common. These cases need a special treatment. However, definitions of TLS and TLQS can be given that encompass not only these but also twofold twins: One speaks of twinning by Twin Lattice Symmetry (TLS) if $\mathbf{L}_{1}$ and $\mathbf{L}_{2}$ have three non-coplanar vectors in common and of twinning by Twin Lattice Quasi Symmetry (TLQS) if two triples of non-coplanar vectors exist in $\mathbf{L}_{1}$ and $\mathbf{L}_{2}$, respectively, that are only approximately equal. This definition extends the one originally given by Donnay and Donnay (1974) by taking account of the zero-obliquity twins by (reticular) pseudo merohedry occurring in case of manifold twins. The cells spanned by the two triples then have the same volume and shape but different orientation.

\subsection{Twin obliquity}

Let us indicate with $\left[u^{\prime} v^{\prime} w^{\prime}\right]$ the direction exactly perpendicular to $(h k l)$, and with $\left(h^{\prime} k^{\prime} l^{\prime}\right)$ the plane perpendicular to $[u v w] .\left[u^{\prime} v^{\prime} w^{\prime}\right]$ is thus parallel to the reciprocal lattice vec- 


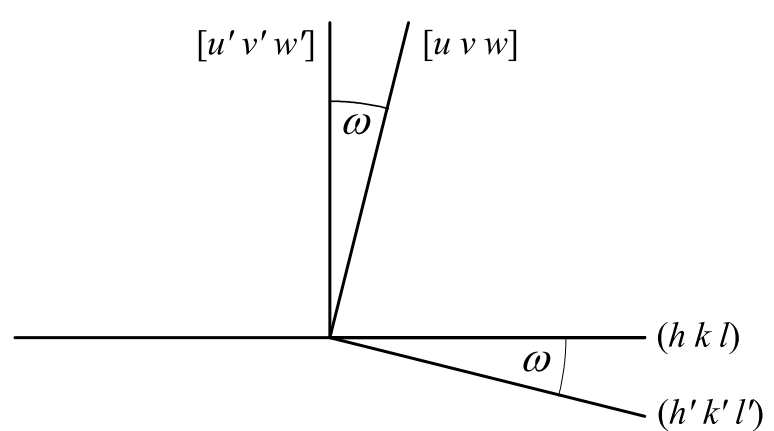

Fig. 2. Definition of the obliquity $\omega$.

tor $[h k l]^{*}$ and $\left(h^{\prime} k^{\prime} l^{\prime}\right)$ is parallel to the reciprocal lattice plane $(u v w)^{*}$. For twofold twins the corresponding primed and unprimed indices coincide in case of TLS. In case of TLQS they do not; moreover, the primed indices are not proportional to integers with low values, their ratios may even be irrational.

The angle between $[u v w]$ and $\left[u^{\prime} v^{\prime} w^{\prime}\right]$ or, which is the same, between $(h k l)$ and $\left(h^{\prime} k^{\prime} l^{\prime}\right)$ is called the obliquity $\omega$ (see Fig. 2).

In geminography we are interested in pairs $[u v w],(h k l)$ with low indices and a small value of $\omega$ (typically $\omega<6^{\circ}$ ). This is because the probability of occurrence of a twin decreases with increasing $\omega$ and with increasing indices of the twin axis/plane, as shown in the next section. Then $[u v w]$ and $(h k l)$ are called quasi-normal; they are normal if $\omega=0$.

The vector in direct space $[u v w]$ has length $L(u v w)$; $\left[u^{\prime} v^{\prime} w^{\prime}\right]$ is parallel to the reciprocal lattice vector $[h k l]^{*}$, whose length is $L^{*}(h k l)$. The obliquity $\omega$ is thus the angle between the vectors $[u v w]$ and $[h k l]^{*}$; the scalar product between these two vectors is

$L(u v w) L^{*}(h k l) \cos \omega=\langle u v w \mid h k l\rangle=u h+v k+w l$,

where \langle| stands for a $1 \times 3$ row matrix and |\rangle for a $3 \times 1$ column matrix.

It follows that

$\cos \omega=\frac{u h+v k+w l}{L(u v w) L^{*}(h k l)}$ where $\quad L(u v w)=\langle u v w|\mathbf{G}| u v w\rangle^{1 / 2} \quad$ and $\quad \mathrm{L}^{*}(h k l)$ $=\left\langle h k l\left|\mathbf{G}^{*}\right| h k l\right\rangle{ }^{1 / 2}, \mathbf{G}$ and $\mathbf{G}^{*}$ being the metric tensors in direct and reciprocal space, respectively. Notice that $\mathbf{G}^{*}=\mathbf{G}^{-1}$ (and thus $\mathbf{G}=\mathbf{G}^{*-1}$ ) and that the matrix representation of the metric tensor is symmetric and coincides thus with its transpose $\left(\mathbf{G}=\mathbf{G}^{\mathrm{T}}, \mathbf{G}^{*}=\mathbf{G}^{* \mathrm{~T}}\right)$. If the basis is primitive and if $p, q, r$ are coprime integers (i.e. integers without common divisor) then $L(p q r)$ is the length of a smallest lattice vector in its direction and $1 / L^{*}(p q r)$ the distance of neighbouring lattice planes.

For triclinic (anorthic) crystals the square of $L(u v w)$ can be expressed as follows in terms of the lattice parameters $a, b, c, \alpha, \beta, \gamma$ :

$$
\begin{aligned}
L^{2}(u v w)= & u^{2} a^{2}+v^{2} b^{2}+w^{2} c^{2} \\
& +2 v w b c \cos \alpha+2 u w a c \cos \beta+2 u v a b \cos \gamma .
\end{aligned}
$$

The square of $L^{*}(h k l)$ can be analogously expressed in terms of the parameters $a^{*}, b^{*}, c^{*}, \alpha^{*}, \beta^{*}, \gamma^{*}$, which characterize the reciprocal lattice. It can be expressed also in terms of the parameters of the direct lattice (see e.g. p. 72 in Giacovazzo, 2002):

$$
\begin{aligned}
& L^{* 2}(h k l) \\
& =\left(1-\cos ^{2} \alpha-\cos ^{2} \beta-\cos ^{2} \gamma+2 \cos \alpha \cos \beta \cos \gamma\right)^{-1} \\
& \quad \cdot\left[\frac{h^{2}}{a^{2}} \sin ^{2} \alpha+\frac{k^{2}}{b^{2}} \sin ^{2} \beta+\frac{l^{2}}{c^{2}} \sin ^{2} \gamma\right. \\
& \quad+\frac{2 k l}{b c}(\cos \beta \cos \gamma-\cos \alpha)+\frac{2 l h}{c a}(\cos \gamma \cos \alpha-\cos \beta) \\
& \left.\quad+\frac{2 h k}{a b}(\cos \alpha \cos \beta-\cos \gamma)\right] .
\end{aligned}
$$

For crystals of higher symmetry the expressions for $L^{2}(u v w)$ and $L^{* 2}(h k l)$ simplify and give rise to the expressions for $\cos \omega$ shown in Table 3 if a conventional coordinate system is used.

\begin{tabular}{|c|c|c|c|}
\hline $\begin{array}{l}\text { Lattice } \\
\text { type }\end{array}$ & $\begin{array}{l}\text { Coordinate } \\
\text { system }\end{array}$ & $\begin{array}{l}\text { Lattice } \\
\text { parameters }\end{array}$ & $\cos \omega$ \\
\hline$c P, c I, c F$ & Cubic & $\begin{array}{l}a=b=c \\
\alpha=\beta=\gamma=90^{\circ}\end{array}$ & $\frac{u h+v k+w l}{\sqrt{u^{2}+v^{2}+w^{2}} \sqrt{h^{2}+k^{2}+l^{2}}}$ \\
\hline$h P, h R$ & Hexagonal & $\begin{array}{l}a=b, c \\
\alpha=\beta=90^{\circ}, \gamma=120^{\circ}\end{array}$ & $\frac{u h+v k+w l}{\sqrt{\left(u^{2}-u v+v^{2}\right) a^{2}+w^{2} c^{2}} \sqrt{\frac{4}{3}\left(h^{2}+h k+k^{2}\right) a^{-2}+l^{2} c^{-2}}}$ \\
\hline$t P, t I$ & Tetragonal & $\begin{array}{l}a=b, c \\
\alpha=\beta=\gamma=90^{\circ}\end{array}$ & $\frac{u h+v k+w l}{\sqrt{\left(u^{2}+v^{2}\right) a^{2}+w^{2} c^{2}} \sqrt{\left(h^{2}+k^{2}\right) a^{-2}+l^{2} c^{-2}}}$ \\
\hline $\begin{array}{l}o P, o C \\
o I, o F\end{array}$ & Orthorhombic & $\begin{array}{l}a, b, c \\
\alpha=\beta=\gamma=90^{\circ}\end{array}$ & $\frac{u h+v k+w l}{\sqrt{u^{2} a^{2}+v^{2} b^{2}+w^{2} c^{2}} \sqrt{h^{2} a^{-2}+k^{2} b^{-2}+l^{2} c^{-2}}}$ \\
\hline$m P, m I$ & Monoclinic & $\begin{array}{l}a, b, c, \beta \\
\alpha=\gamma=90^{\circ}\end{array}$ & $\frac{u h+v k+w l}{\sqrt{u^{2} a^{2}+v^{2} b^{2}+w^{2} c^{2}+2 u w a c \cos \beta} \sqrt{\frac{1}{\sin ^{2} \beta}\left(\frac{h^{2}}{a^{2}}+\frac{k^{2} \sin ^{2} \beta}{b^{2}}+\frac{l^{2}}{c^{2}}-\frac{2 h l \cos \beta}{a c}\right)}}$ \\
\hline
\end{tabular}

The indices $u^{\prime} v^{\prime} w^{\prime}$ of the direction normal to the lattice plane $(h k l)$, and the indices $h^{\prime} k^{\prime} l^{\prime}$ of the plane normal to the lattice row $[u v w]$, can easily be calculated using the metric tensor:

$$
\left|u^{\prime} v^{\prime} w^{\prime}\right\rangle=\mathbf{G}^{*}|h k l\rangle \quad \text { and } \quad\left|h^{\prime} k^{\prime} l^{\prime}\right\rangle=\mathbf{G}|u v w\rangle .
$$

Table 3. The twin obliquity $\omega$ for crystals with at least monoclinic symmetry. 


\subsection{Twin index}

We defined the twin index (or multiplicity) $n$ as the volume ratio of primitive cells in the twin lattice and crystal lattice, $n=V_{\mathrm{T}} / V$.

For TLS three vectors that span a primitive cell of the twin lattice are simultaneously vectors of $\mathbf{L}_{1}$ and of $\mathbf{L}_{2}$. The twin lattice $\mathbf{L}_{\mathrm{T}}$ is the common sublattice of $\mathbf{L}_{1}$ and $\mathbf{L}_{2}$. The density of its lattice points is $n$-times smaller than the density of nodes in $\mathbf{L}_{1}$ or $\mathbf{L}_{2}$.

For TLQS there is a cell in $\mathbf{L}_{1}$ that nearly coincides with a cell in $\mathbf{L}_{2}$ of the same volume and shape. The twin index $n$ is the ratio of its volume $V_{\mathrm{T}}$ to the volume $V$ of a primitive cell of $\mathbf{L}_{1}$ or $\mathbf{L}_{2}$.

A (not necessarily primitive) cell of the twin lattice is spanned:

1. in the case of a twin axis $[u v w]$, by a smallest lattice vector in the direction of the twin axis and a smallest mesh of the net $(h k l)$ that is normal or quasi-normal to it;

2 . in the case of a twin plane $(h k l)$, by a smallest mesh of the net in the twin plane and a smallest lattice vector $[u v w]$ that is normal or quasi-normal to it.

For twofold twins with $\omega=0$ (TLS), the same orientation relation exists between $\mathbf{L}_{1}$ and $\mathbf{L}_{2}$, regardless whether the two twin individuals are related by a $180^{\circ}$ rotation about $[u v w]$ or a reflection in $(h k l)$. The formula for the twin index is therefore the same for rotation twins and reflection twins. For TLQS, instead, although the twin index is defined in the same way once the choice of the pair twin axis/twin plane has been made:

- the relative orientation of $\mathbf{L}_{1}$ and $\mathbf{L}_{2}$ is no longer exactly the same for rotation twins and reflection twins,

- the parallelohedra defined by $(h k l)$ and $[u v w]$ in $\mathbf{L}_{1}$ and $\mathbf{L}_{2}$ no longer coincide exactly.

An example is provided by the 'albite' and 'pericline' twin laws in triclinic feldspars, discussed in Section 4.2.1.

For a given twin plane two different quasi-normal axes $\left[u^{\prime} v^{\prime} w^{\prime}\right]$ and $\left[u^{\prime \prime} v^{\prime \prime} w^{\prime \prime}\right]$ may sometimes be chosen, corresponding to higher indices but lower obliquity or to lower indices but higher obliquity. Similarly, for a given twin axis $[u v w]$, a quasi-normal plane $(h k l)$ is not uniquely defined.

A given choice of a (quasi-)normal pair $(h k l) /[u v w]$ determines $n$ uniquely for both TLS and TLQS and for both twofold and manifold twins. In order to compute $n$ we consider the sublattice $\mathbf{L}_{\mathrm{T}}$ of $\mathbf{L}_{1}$ that coincides exactly (for TLS) or approximately (for TLQS) with a sublattice of $\mathbf{L}_{2}$.

a) A primitive basis is chosen for $\mathbf{L}_{1}$ :

Take $h, k$ and $l$ to be coprime integers, similarly for $u, v$ and $w$. The parallelohedron having as two of its six faces a smallest mesh in $(h k l)$ and as four of its 12 edges the vector $[u v w]$ is a cell of $\mathbf{L}_{T}$ with a volume that is $S=|h u+k v+l w|$ times larger than the volume $V$ of a primitive cell of $\mathbf{L}_{1}$. Because $u, v$ and $w$ are coprime, $[u v w]$ is the shortest lattice vector in its direction. Nevertheless, the parallelohedron is not always a primitive cell of $\mathbf{L}_{\mathrm{T}}$. It may contain an additional node of $\mathbf{L}_{\mathrm{T}}$ in its centre or in the centres of a pair of opposite faces, (quasi-)normal to $(h k l)$. This is the case if and only if $S$ is even. The twin index $n=V_{\mathrm{T}} / V$ therefore satisfies

$$
n=S \text { if } S \text { is odd, } \quad n=S / 2 \text { if } n \text { is even. }
$$

b) A centred basis is chosen for $\mathbf{L}_{1}$ :

The formula for the twin index is modified if $(h k l)$ and $[u v w]$ do not refer to a primitive basis for $\mathbf{L}_{1}$. In fact, the direct lattice then contains also vectors $[u v w]$ whose components are not all integral; on the other hand, not all $(h k l)$ with integral components are vectors of the reciprocal lattice. Taking ( $h k l)$ as a triple of integers without common divisor and similarly for $[u v w]$, the results shown in Table 4 are obtained.

A alternative approach has been taken by E. Koch (2004), who, also in case of a non-primitive basis, chooses $u, v, w$ to be the components of a shortest lattice vector in the direction $[u v w]$ and $h, k, l$ to be smallest integers defining a vector of the reciprocal lattice in the direction $(h k l)$, i.e. $u, v, w$ may not be integers, $h, k, l$ not coprime. The formula given above for a primitive basis then holds also if a centred basis is chosen.

\subsection{The twin lattice of twofold twins in case of TLQS}

In case of TLQS the twin lattice of twofold twins undergoes a slight deviation at the composition surface (Friedel, 1904, 1926; Donnay, 1940). Let $\mathbf{L}_{\mathrm{S} 1}$ and $\mathbf{L}_{\mathrm{S} 2}$ be the sublattices with multiplicity $n$ defined by $(h k l)$ and $[u v w]$ in $\mathbf{L}_{1}$ and $\mathbf{L}_{2}$, respectively. $\mathbf{L}_{\mathrm{S} 1}$ and $\mathbf{L}_{\mathrm{S} 2}$ coincide for TLS and form the twin lattice $\mathbf{L}_{\mathrm{T}}$. They no longer coincide for TLQS but have slightly different orientations.

If the twin law can be expressed by giving a twin mirror plane $(h k l)$, then the two individuals have all translations in this plane in common. In the lattice planes parallel to $(h k l)$ the nodes are not restored exactly in case of TLQS. If, however, the nodes are nearly restored in the $n$ th lattice plane parallel to $(h k l)$ one says that the twin index is $n$. This definition shows that the twin index is not always defined uniquely in the case of TLQS. In the following examples we shall see that ambiguities may exist when two alternative descriptions of a TLQS twin exist, corresponding to lower obliquity/higher index and lower index/higher obliquity, respectively.

\subsection{Derivation of the pseudo-normal axis from the twin plane}

As shown above, the direction perpendicular to a lattice plane $(h k l)$, where $h, k$ and $l$ are coprime integers, has indices $\left[u^{\prime} v^{\prime} w^{\prime}\right]$ that are easily computed on the basis of the transformation

$$
\left|u^{\prime} v^{\prime} w^{\prime}\right\rangle=\mathbf{G}^{*}|h k l\rangle
$$

where $\mathbf{G}^{*}$ is the reciprocal metric tensor. Let $(h k l)$ be the twin plane. If the mirror reflection in $(h k l)$ is a symmetry element of $\mathbf{L}_{1}$, then the twinning is merohedric, otherwise not.

The indices $u^{\prime}, v^{\prime}$ and $w^{\prime}$ as obtained from the above equation in general are not integer. If $s_{1}=u^{\prime} / v^{\prime}$ and $s_{2}=u^{\prime} / w^{\prime}$ are rational, then also $s_{2} / s_{1}=v^{\prime} / w^{\prime}$ is rational and the direction perpendicular to $(h k l)$ is a rational di- 
Table 4. Computation of the twin index as a function of $S=|h u+k v+l w|$ and of the centring of the individual lattice (see Friedel, 1926; Donnay and Donnay, 1959).

\begin{tabular}{|c|c|c|c|c|}
\hline $\begin{array}{l}\text { Lattice } \\
\text { centring }\end{array}$ & $\begin{array}{l}\text { Condition on } h, k, l \\
(u, v, w \text { for } F \text {-centring) }\end{array}$ & $\begin{array}{l}\text { Condition on } u, v, w \\
(h, k, l \text { for } F \text {-centring) }\end{array}$ & $\begin{array}{l}\text { Condition on } \\
S=|h u+k v+l w|\end{array}$ & $\begin{array}{l}\text { Twin index } \\
n\end{array}$ \\
\hline \multirow[t]{2}{*}{$P$} & none & none & $S$ odd & $n=S$ \\
\hline & & & $S$ even & $n=S / 2$ \\
\hline \multirow[t]{5}{*}{$C$} & $h+k$ odd & none & none & $n=S$ \\
\hline & $h+k$ even & $u+v$ and $w$ not both even & $S$ odd & $n=S$ \\
\hline & & & $S$ even & $n=S / 2$ \\
\hline & & $u+v$ and $w$ both even & $S / 2$ odd & $n=S / 2$ \\
\hline & & & $S / 2$ even & $n=S / 4$ \\
\hline \multirow[t]{5}{*}{$B$} & $h+l$ odd & none & none & $n=S$ \\
\hline & $h+l$ even & $u+w$ and $v$ not both even & $S$ odd & $n=S$ \\
\hline & & & $S$ even & $n=S / 2$ \\
\hline & & $u+w$ and $v$ both even & $S / 2$ odd & $n=S / 2$ \\
\hline & & & $S / 2$ even & $n=S / 4$ \\
\hline \multirow[t]{5}{*}{$A$} & $k+l$ odd & none & none & $n=S$ \\
\hline & $k+l$ even & $v+w$ and $u$ not both even & $S$ odd & $n=S$ \\
\hline & & & $S$ even & $n=S / 2$ \\
\hline & & $v+w$ and $u$ both even & $S / 2$ odd & $n=S / 2$ \\
\hline & & & $S / 2$ even & $n=S / 4$ \\
\hline \multirow[t]{5}{*}{$I$} & $h+k+l$ odd & none & none & $n=S$ \\
\hline & $h+k+l$ even & $u, v, w$ not all odd & $S$ odd & $n=S$ \\
\hline & & & $S$ even & $n=S / 2$ \\
\hline & & $u, v, w$ all odd & $S / 2$ odd & $n=S / 2$ \\
\hline & & & $S / 2$ even & $n=S / 4$ \\
\hline \multirow[t]{5}{*}{$F$} & $u+v+w$ odd & none & none & $n=S$ \\
\hline & $u+v+w$ even & $\mathrm{h}, k, l$ not all odd & $S$ odd & $n=S$ \\
\hline & & & $S$ even & $n=S / 2$ \\
\hline & & $h, k, l$ all odd & $S / 2$ odd & $n=S / 2$ \\
\hline & & & $S / 2$ even & $n=S / 4$ \\
\hline
\end{tabular}

rection, whose indices can easily be transformed to coprime integers. If the result does not consist of too large values of the computed indices, the direction $\left[u^{\prime} v^{\prime} w^{\prime}\right]$ is called rational and the twin has zero obliquity (TLS).

If instead either $s_{1}$ or $s_{2}$ (or both) are not rational, or if the values of the indices are too large to correspond to a rational direction, the twin has non-zero obliquity (TLQS). This obliquity is defined with respect to a rational direction $\left[u_{\mathrm{T}} v_{\mathrm{T}} w_{\mathrm{T}}\right]$ close to $\left[u^{\prime} v^{\prime} w^{\prime}\right]$. This direction in general is not unique, in fact, one may often choose two directions, $\left[u_{\mathrm{T}} v_{\mathrm{T}} w_{\mathrm{T}}\right]_{\mathrm{A}}$ and $\left[u_{\mathrm{T}} v_{\mathrm{T}} w_{\mathrm{T}}\right]_{\mathrm{B}}$, which correspond to different obliquities $\left(\omega_{\mathrm{A}}\right.$ ad $\left.\omega_{\mathrm{B}}\right)$ and twin indices $\left(n_{\mathrm{A}}\right.$ and $\left.n_{\mathrm{B}}\right)$, with $\omega_{\mathrm{A}}>\omega_{\mathrm{B}}$ but $n_{\mathrm{A}}<n_{\mathrm{B}}$. When the indices $u_{\mathrm{T}} v_{\mathrm{T}} w_{\mathrm{T}}$ for both directions are comparably small, the description of the twin may not be unique.

Example. The mineral epistolite,

$\mathrm{Na}_{2} \mathrm{Ti}_{2} \mathrm{Nb}_{2}\left(\mathrm{Si}_{2} \mathrm{O}_{7}\right)_{2}(\mathrm{OH})_{4} \cdot 2\left(\mathrm{H}_{2} \mathrm{O}\right)$, space group $P \overline{1}$, $a=5.460 \AA, b=7.170 \AA, c=12.041 \AA, \quad \alpha=103.63^{\circ}$, $\beta=96.01^{\circ}, \gamma=89.98^{\circ}$ (Sokolova and Hawthorne, 2004), gives a twin with twin plane (001). The direction perpen- dicular to this plane is:

$$
\begin{aligned}
& \mathbf{G}^{*}|h k l\rangle=\left[\begin{array}{lll}
0.03393 & 0.00061 & 0.00170 \\
0.00061 & 0.02061 & 0.00292 \\
0.00170 & 0.00292 & 0.00739
\end{array}\right]|001\rangle \\
& =\left|\begin{array}{lll}
0.00170 & 0.00292 & 0.00739
\end{array}\right| \\
& \approx|1.16 \ldots 2 \quad 5.06 \ldots\rangle=\left|u^{\prime} v^{\prime} w^{\prime}\right\rangle .
\end{aligned}
$$

The rational direction closest to this one is [125], which makes with it an angle of $0.84^{\circ}$. The calculation of the twin index is easy: $n=S=|h u+k v+l w|=5$. However, not far from [125] is the direction [124], which makes a higher angle with $\left[u^{\prime} v^{\prime} w^{\prime}\right], 3.71^{\circ}$, but defines a lower twin index: $S=|h u+k v+l w|=4$ and the index is $n=S /$ $2=2$, where we used the fact that the basis of the crystal lattice is primitive. Because this twin is a growth twin, and because in growth twins the obliquity normally plays a secondary role compared to the twin index (Friedel, 1923), this twin law is usually described as $(001) /[124]$. Nevertheless, $(001) /[125]$ is a possible alternative description. 


\subsection{Derivation of the cell of the sublattice for non-merohedric twins}

A twin axis (plane) is a (pseudo)-symmetry axis (plane) for $\mathbf{L}_{\mathrm{T}}$. It follows that in case of TLS the point symmetry of $\mathbf{L}_{\mathrm{T}}$ is at least $2 / \mathrm{m}$; in case of TLQS, this is the minimal pseudo-symmetry of $\mathbf{L}_{\mathrm{T}}$. To find the conventional cell of $\mathbf{L}_{T}$ we can start taking the twin plane as (010) or the twin axis as [010]: if $\mathbf{L}_{\mathrm{T}}$ turns out to have a point symmetry higher than $2 / m$, a suitable transformation is then applied. The starting cell of $\mathbf{L}_{\mathrm{T}}$ is defined either by the highestsymmetry mesh in the twin plane and its (quasi) normal axis or by the twin axis and the highest-symmetry mesh in its (quasi) normal plane. First of all, two shortest non-collinear vectors $\boldsymbol{a}=[u v w]_{1}$ and $\boldsymbol{b}=[u v w]_{2}$ in the $(h k l)$ plane are determined. They must obey the condition $h u_{j}+k v_{j}+l w_{j}=0$. Let us indicate their lengths with $a$ and $b$, and the angle $\boldsymbol{a} \wedge \boldsymbol{b}$ as $\varphi$. We may choose [uvw $]_{1}$ and $[u v w]_{2}$ such that $a \leq b$ and $0 \leq 2 \boldsymbol{a} \cdot \boldsymbol{b} \leq a^{2}$. The conventional mesh is obtained in the following way:

- if $a<b$ and $0<2 \boldsymbol{a} \cdot \boldsymbol{b}<a^{2}$, the smallest mesh coincides with the conventional mesh, its type is $m p$

- if $a<b$ and $\boldsymbol{a} \cdot \boldsymbol{b}=0$, i.e. $\varphi=90^{\circ}$, the smallest mesh coincides with the conventional mesh, its type is $o p$;

- if $a=b$ and $\boldsymbol{a} \cdot \boldsymbol{b}=0$, i.e. $\varphi=90^{\circ}$, the smallest mesh coincides with the conventional mesh, its type is $t p$;

- if $a=b$ and $2 \boldsymbol{a} \cdot \boldsymbol{b}=a^{2}$, i.e. $\varphi=60^{\circ}$, the smallest mesh coincides with the conventional mesh, its type is $h p$;

- if $a=b$ and $0<2 \boldsymbol{a} \cdot \boldsymbol{b}<a^{2}$, i.e. $60^{\circ}<\varphi<90^{\circ}$, or if $a<b$ and $2 \boldsymbol{a} \cdot \boldsymbol{b}=a^{2}$, i.e. $2 \cos \varphi=a / b$, the smallest mesh does not coincide with the conventional mesh; this one has type $o c$ and is obtained via the axial transformation $\boldsymbol{a}^{\prime}=\boldsymbol{a}-\boldsymbol{b}, \boldsymbol{b}^{\prime}=\boldsymbol{a}+\boldsymbol{b}$ in the first case and $\boldsymbol{a}^{\prime}=\boldsymbol{a}, \boldsymbol{b}^{\prime}=2 \boldsymbol{b}-\boldsymbol{a}$ in the second; $1<b^{\prime} / a^{\prime}<\sqrt{3}$ in the first case, $b^{\prime} / a^{\prime}>\sqrt{3}$ in the second.

The transformation from $\mathbf{L}_{\mathrm{I}}$, the lattice of the individual, to $\mathbf{L}_{\mathrm{T}}$ is easily obtained via the corresponding metric tensors, where the transformation matrix is built on the two directions $[u v w]_{1}$ and $[u v w]_{2}$ and on the (quasi) normal direction $[u v w]_{\mathrm{T}}$. Keeping in mind that the directions in direct space are normally given by their contravariant components and the basis vectors by their covariant components, the transformation matrix is composed by the indices of the three directions above as columns.

$$
\mathbf{G}_{\mathrm{T}}=\mathbf{U}^{\mathrm{t}} \mathbf{G}_{\mathrm{I}} \mathbf{U}, \quad \mathbf{U}=\left[\begin{array}{ccc}
u_{1} & u_{\mathrm{T}} & u_{2} \\
v_{1} & v_{\mathrm{T}} & v_{2} \\
w_{1} & w_{\mathrm{T}} & w_{2}
\end{array}\right] .
$$

Here $\mathbf{G}_{\mathrm{T}}$ and $\mathbf{G}_{\mathrm{I}}$ are the metric tensors of $\mathbf{L}_{\mathrm{T}}$ and of $\mathbf{L}_{\mathrm{I}}$ respectively, and $\mathbf{U}^{t}$ is the transpose of $\mathbf{U}$. The result may however not yet coincide with the conventional cell of $\mathbf{L}_{\mathrm{T}}$ and a further transformation may be needed. In fact, the cell obtained above may have a non-conventional centring.

The mesh of $\mathbf{L}_{\mathrm{T}}$ defined by two directions $[u v w]_{\mathrm{T}}$ and $[u v w]_{1}$, may contain several lattice nodes in its interior, and the same holds for the mesh defined by two directions $[u v w]_{\mathrm{T}}$ and $[u v w]_{2}$; in both cases only one of these nodes can be restored (exactly, in case of TLS; approximately, in case of TLQS) by the twin operation: the node at the centre of the mesh. In the following we restrict our attention to the case where a primitive basis has been chosen for $\mathbf{L}_{\mathrm{I}}$. Then each point of $\mathbf{L}_{\mathrm{I}}$ has integral coordinates and to each triple of integers corresponds a point of $\mathbf{L}_{\mathrm{I}}$. A node centres a mesh if either $u_{1}+u_{\mathrm{T}}, v_{1}+v_{\mathrm{T}}, w_{1}+w_{\mathrm{T}}$ are all $0(\bmod 2)$ or if $u_{\mathrm{T}}+u_{2}, v_{\mathrm{T}}+v_{2}, w_{\mathrm{T}}+w_{2}$ are all $0(\bmod$ 2 ). Besides, also the $I$-centring has to be checked; it corresponds to the condition that $u_{1}+u_{2}+u_{\mathrm{T}}, v_{1}+v_{2}+v_{\mathrm{T}}$, $w_{1}+w_{2}+w_{\mathrm{T}}$ are all $0(\bmod 2)$. If $\mathbf{U}^{\prime}$ is the matrix transforming the cell obtained via the $\mathbf{U}$ matrix into the conventional cell, the global transformation $\mathbf{L}_{\mathrm{I}} \rightarrow \mathbf{L}_{\mathrm{T}}$ is given by the matrix product $\mathbf{U U}^{\prime}$.

Example. The (001)/[124] description of the epistolite twin above gives rise to a non-primitive triclinic, pseudomonoclinic cell. In fact, two shortest non-collinear lattice vectors in (001) are [100] and [010]. The mesh defined by the directions [100] and [124] is centred because $u_{1}+u_{2}$, $v_{1}+v_{2}$ and $w_{1}+w_{2}$ are all even. The transformation from $\mathbf{L}_{\mathrm{I}}$ to $\mathbf{L}_{\mathrm{T}}$ is obtained by:

$$
\left\langle\left.\boldsymbol{a b c}\right|_{I}\left[\begin{array}{ccc}
0 & 1 & 1 \\
1 & 2 & 0 \\
0 & 4 & 0
\end{array}\right]=\left\langle\left.\boldsymbol{a b c}\right|_{\mathrm{T}}\right.\right.
$$

with cell parameters $a=7.170 \AA ; \quad b=46.634 \AA$, $c=5.460 \AA, \alpha=89.61^{\circ}, \beta=89.98^{\circ} \gamma=85.17^{\circ}$. In this setting the cell is $A$-centred.

The alternative description $(001) /[125]$ corresponds to a primitive cell for $\mathbf{L}_{\mathrm{T}}$. In fact the meshes of the cell of $\mathbf{L}_{\mathrm{T}}$ are defined by the three pairs of directions formed by [010], [100] and [125]

- the mesh defined by the directions [010] and [100] is not centred because $u_{1}+u_{2}$ and $v_{1}+v_{2}$ are odd;

- the mesh defined by the directions [010] and [125] is not centred because $u_{1}+u_{2}, v_{1}+v_{2}$ and $w_{1}+w_{2}$ are all odd;

- the mesh defined by the directions [100] and [ [ 12 5] is not centred because $w_{1}+w_{2}$ is odd.

The last check to perform is towards the $I$-centring. This corresponds to the centring of the mesh defined by the vector sum of the three directions $[u v w]_{1},[u v w]_{2}$ and $[u v w]_{\mathrm{T}}$. The results are odd for $v$ and $w$ and thus the cell of $\mathbf{L}_{T}$ defined by $(h k l)$ and $[u v w]$ is $P$. The transformation matrix from $\mathbf{L}_{\mathrm{I}}$ to $\mathbf{L}_{\mathrm{T}}$ is:

$$
\left\langle\left.\boldsymbol{a b c}\right|_{I}\left[\begin{array}{ccc}
0 & 1 & 1 \\
1 & 2 & 0 \\
0 & 5 & 0
\end{array}\right]=\left\langle\left.\boldsymbol{a b c}\right|_{\mathrm{T}} .\right.\right.
$$

The result is a triclinic primitive, pseudo-orthorhombic cell with cell parameters $a=7.170 \AA ; \quad b=58.176 \AA$, $c=5.460 \AA, \alpha=90.83^{\circ}, \beta=89.98^{\circ} \gamma=89.85^{\circ}$.

\section{Classification of twins in terms of their merohedricity}

\subsection{Friedel's classification of twins}

Friedel (1904) distinguished four types of twins according to whether the twin index is 1 or larger and the obliquity 0 or larger, as shown in Table 5. 
Table 5. The four types of twinning distinguished by Friedel (1904).

\begin{tabular}{lll}
\hline & TLS $(\omega=0)$ & TLQS $(\omega>0)$ \\
\hline$n=1$ & Twins by merohedry & Twins by \\
& $=$ merohedric twins & pseudo-merohedry \\
$n>1$ & Twins by reticular & Twins by reticular \\
& merohedry & pseudo-merohedry \\
\hline
\end{tabular}

Although the obliquity distinguishes between TLS and TLQS only in the case of twofold twins, Friedel (1926, p. 442) described also the well-known $90^{\circ}$-rotation twin in leucite as an example of a twin by pseudo-merohedry, without mentioning that $\omega=0$ in this case, which will be discussed in Section 4.2.1.

Friedel considered twinning to be the more probable, the smaller $n$ and $\omega$. He proposed limits that are often satisfied by observed twins: $n<6, \omega<6^{\circ}$ and mentioned that his ideas were based on the work of his teacher Mallard. We shall make the following distinction:

"Mallard's law" (Friedel, 1904, 1926; Donnay, 1940): The relative orientation of $\mathbf{L}_{1}$ and $\mathbf{L}_{2}$ can be described either by a mirror reflection in a lattice plane $(h k l)$ or a crystallographic rotation about a lattice row $[u v w]$.

\section{"Mallard's criterion":}

For reflection twins with twin plane $(h k l)$ there exists a (quasi-)normal lattice row $[u v w]$ and for rotation twins with twin axis $[u v w]$ a (quasi-)normal lattice plane $(h k l)$ such that $n<6$ and $\omega<6^{\circ}$.

"Mallard's law" is satisfied for most of the known twins. Also "Mallard's criterion" is usually satisfied; for many crystalline substances the most frequently observed twin laws have the smallest values of $n$.

\subsection{Examples}

We already discussed the Dauphiné, Brazil and combined twins in $\alpha$-quartz as examples of twins by merohedry. Let us now give examples of the other three types.

\subsubsection{Twins by pseudo-merohedry}

Triclinic feldspars belong to the holohedral crystal class $\overline{1}$. They show strong pseudosymmetries to the monoclinic feldspar structures, i.e. (010) and [010] are quasinormal. Possible twins are therefore the reflection twin with twin plane (010) and the rotation twin with twin axis [010]. Both have been observed and have been called "reciprocal twins" (Mügge, 1898) or "corresponding twins" (Friedel, 1904; 1926). The respective twin laws are known as Albite law (010) and Pericline law [010].

Leucite $\left(\mathrm{KAlSi}_{2} \mathrm{O}_{6}\right)$ is cubic with space-group type $I a \overline{3} d$ at high temperature and tetragonal with space-group type $I 4_{1} / a$ and axial ratio $c / a=1.050$ at room temperature. (Pies and Weiss, 1985) A leucite crystal grown at high temperature undergoes twinning when it transforms into the tetragonal phase. The relative orientation of the lattices of two adjacent individuals can be described by a $90^{\circ}$ rotation about one of the 4-fold symmetry axes of the cubic phase that is lost in the phase transition (pseudo-fourfold axis for the individual). The plane perpendicular to the twin rotation axis is a lattice plane: in fact, in a tetragonal lattice the lattice plane $(h k 0)$ is perpendicular to the lattice row [hk0]. It follows that the obliquity $\omega$ is zero, and that the deviation from TLS is characterized in this case by the linear deviation of cla from 1, not by the angular deviation of $\omega$ from 0 .

\subsubsection{Twins by reticular merohedry}

Corundum $\mathrm{Al}_{2} \mathrm{O}_{3}$ and calcite $\mathrm{CaCO}_{3}$ crystallize in space group type $R \overline{3} c$, i.e. they are holohedral. They often show growth twinning with a 2 -fold rotation about [001] as twin operation. The elements of the point group symmetry $\mathscr{H}=\overline{3} 2 / m$ of these crystals are left invariant by the twin operation. The composite symmetry is therefore $\mathscr{K}=6^{\prime}$ / $m^{\prime} 2 / m 2^{\prime} / m^{\prime}$. The twin lattice $\mathbf{L}_{\mathrm{T}}$ is the primitive hexagonal sublattice of $h R$, i.e. $n=3$.

This law is closely connected with the spinel law in holohedral cubic crystals, which can be described by a 2fold twin rotation about one of the four 3-fold axes of a cubic crystal. In fact, all the three Bravais types of cubic lattices, $c P, c I$ and $c F$ are special cases of the rhombohedral lattice $h R$, as shown in Fig. 3.

$\mathscr{H}=m \overline{3} m$ for holohedral cubic crystals. A 2-fold rotation with twin axis [111] leaves only this 3-fold axis and the 2 -fold axes perpendicular to it invariant, i.e. $\mathscr{H}^{*}=\overline{3}$ $2 / m 1$, which has 12 group elements and $\mathscr{K}^{*}=6^{\prime} / m^{\prime} 2 / m$ $2^{\prime} / m^{\prime}$ with 24 elements. The spinel law therefore can be expressed by 12 different twin operations. For hemihedral cubic crystals (point groups $432, \overline{4} 3 m$ and $m \overline{3}$ ) the 12 operations describing the spinel law for cubic holohedral crystals split into two sets of 6 , each set describing a different law. E.g. in the case of 432, one law can be represented by a 2-fold rotation with twin axis [111], the other by a reflection with twin plane (111). $\mathscr{H}^{*}=321$ in both cases, $\mathscr{K}^{*}=6^{\prime} 22^{\prime}$ for the first law, $\mathscr{K}^{*}=\overline{6}^{\prime} 2 m^{\prime}$ for the second. The two individuals have the same handedness in the first case and opposite handedness in the second. Finally, for the tetartohedral point group 23, the 12 operations split into four sets of 3 operations, each set describing a different twin law (for details see Hahn and Klapper, 2003). What is commonly called the "spinel law" encompasses therefore two (four) different laws for hemihedral (tetartohedral) cubic crystals, although the relative orientation of the lattices of the individuals is the same in both (all four) cases. In the next section we shall encounter an

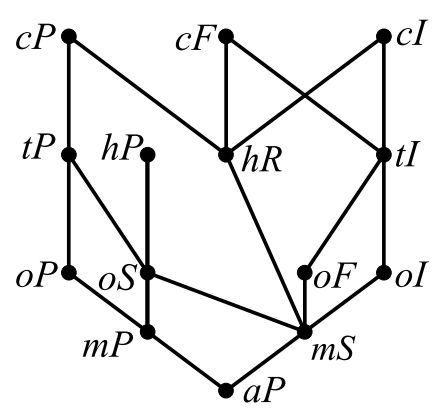

Fig. 3. The relation between the various Bravais types of lattices. The Bravais type at the upper end of a line is a special case with point group of higher order of the one at the lower end. 
analogous situation, concerning the "Japan law" in $\alpha$ quartz.

\subsubsection{Twins by reticular pseudo-merohedry}

The lattices of the two individuals of a Japan twin of $\alpha$ quartz (also known as "La Gardette" twin) are mapped onto each other by a mirror reflection in a $\{11 \overline{2} 2\}$ plane, which coincides with the composition plane. Japan twins are therefore reflection twins in the broad sense, i.e. if only the relative orientation of the two lattices is taken into account. If $\alpha$-quartz were holohedral, there would be only one twin law with this relative orientation of the two lattices. However, Table 1 shows that $\alpha$-quartz with its point group 321 belongs to a tetartohedral crystal class. What is known as the Japan law splits into four different laws if the orientation of the crystal structures of the individuals is taken into account, not only the orientation of their lattices: only one of them (example III in Fig. 4) has $\{11 \overline{2} 2\}$ as twin plane. The others correspond to the combination of this twin operation with the operations for twins with parallel axes: Dauphine (case IV), Brazil (case I) and Leydolt (case II).

This leads to the result given in Table 6 (see Frondel, 1962; Hahn and Klapper, 2003). Notice that [111] lies in the plane $(11 \overline{2} 2)$ and that the axis $\perp(11 \overline{2} 2)$ and the plane $\perp[11 \overline{1}]$ are irrational for irrational values of $(c / a)^{2}$.

In contrast to Brazil, Dauphiné and combined twins, where the two individuals have parallel symmetry axes, these are not parallel in Japan twins. The oriented point groups of the two individuals have no elements in com-

I (R)

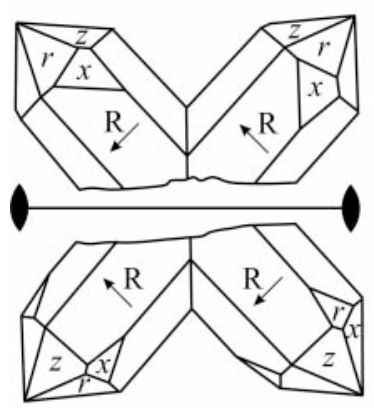

III
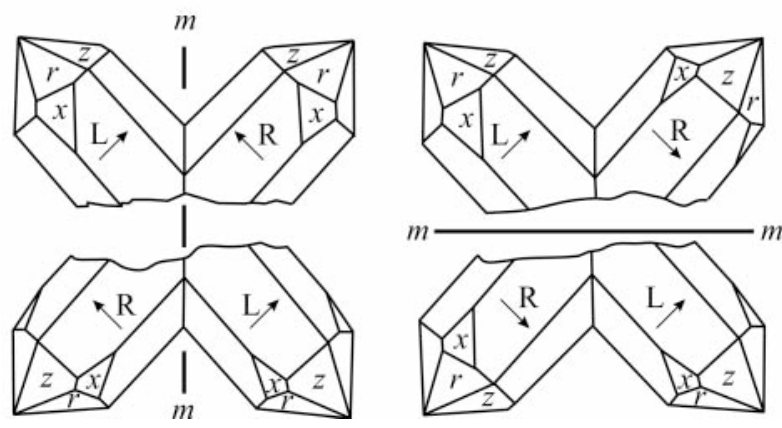

Fig. 4. The four variants of Japanese twins of quartz. The twin elements 2 and $m$ are shown. In an actual twin either the upper or the lower part is realized. R, L: right-, left-handed quartz. The arrow gives the polarity of the twofold axis parallel to the plane of the drawing. The cases $\mathrm{I}(\mathrm{L})$ and $\mathrm{II}(\mathrm{L})$ are not shown. (reproduced from Hahn-Klapper (2003) with the permission of the IUCr).
Table 6. Description of the twin elements for the four kinds of Japan twins (after Frondel, 1962; Hahn and Klapper, 2003).

\begin{tabular}{lll}
\hline & $(h k l)$ rational & {$[u v w]$ rational } \\
\hline Rotation twins & twin axis $\perp(11 \overline{2} 2)$ & twin axis [11 $\overline{1}]$ \\
& Japan I & Japan II \\
Reflection twins & twin plane $(11 \overline{2} 2)$ & twin plane $\perp[11 \overline{1}]$ \\
& Japan III & Japan IV \\
\hline
\end{tabular}

mon, $\mathscr{H}^{*}=1$; each of the four Japan twin laws may be expressed by only one twin operation.

The relative orientation of the lattices of the two individuals is the same for all four cases; also the composition plane is $(11 \overline{2} 2)$ in all four cases: The most convenient description of the relative orientation of the lattices is therefore the one corresponding to case III. The other three cases can be obtained by combining the mirror reflection $(1 \overline{2} 2)$ with the twin operations of the Brazil, Dauphine and combined twin, i.e. $\overline{1}, 2[001]$ or $m[001]$, respectively.

In summary: From the point of view of the relative orientation of the lattices, there is just one kind of Japan twin, the reflection twin with twin plane $(11 \overline{2} 2)$; from the point of view of the relative orientation of the crystal structures, four kinds I-IV have to be distinguished.

A further example of twinning by reticular pseudo-merohedry is the (001) twin in epistolite, which has been considered in connection with the twin lattice.

\subsection{A finer classification of twins}

The definition of syngonic merohedry contains a subtle point, which can be discussed with the help of Table 1 . Consider a crystal with point group $\mathscr{H}$ and let $\mathscr{D}$ be its holohedral supergroup that belongs to the same lattice system. Table 1 shows that in case of point groups belonging to the trigonal crystal system, $\mathscr{D}$ will depend on whether the crystal lattice is of type $h R$ or $h P$. Notice that the point group $\mathscr{H}$ coincides with $\mathscr{D}$ for holohedral crystals. The point group of the crystal lattice $\mathbf{L}, \mathscr{D}(\mathbf{L})$, is always holohedral. Normally, $\mathscr{D}=\mathscr{D}(\mathbf{L})$, but when the crystal has $a c$ cidentally a specialized metric, then $\mathscr{D}(\mathbf{L})$ is a supergroup of $\mathscr{D}$.

When $n>1$ one has to distinguish between $\mathbf{L}_{\mathrm{I}}$, the lattice of the individual, and $\mathbf{L}_{\mathrm{T}}$, the lattice of the twin and thus also between $\mathscr{D}\left(\mathbf{L}_{\mathrm{I}}\right)$ and $\mathscr{D}\left(\mathbf{L}_{\mathrm{T}}\right)$ - which coincide for $n=1$.

Twinning can be classified, on the basis of $\mathscr{H}, \mathscr{D}$, $\mathscr{D}\left(\mathbf{L}_{\mathrm{I}}\right), \mathscr{D}\left(\mathbf{L}_{\mathrm{T}}\right), n$ and $\omega$, into the following categories.

1 Twinning by merohedry: $n=1, \omega=0$. The twin element can be the centre (for non-centrosymmetric crystals), an axis, a plane, as shown in Table 1. Twinning by merohedry is subdivided into the following subcategories.

1.1 Twinning by syngonic merohedry: $\mathscr{H} \subset \mathscr{D}$ and the twin operation belongs to $\mathscr{D}$. When the twin element is the inversion centre (only non centrosymmetric crystals), we speak of class I twins, otherwise of class IIA twins (Catti and Ferraris, 1976; Nespolo and Ferraris, 2000). Two possible subcases exist. 
1.1.1 $\mathscr{D}=\mathscr{D}(\mathbf{L})$. This is the most frequent case, where the lattice does not have a specialized metric.

1.1.2 $\mathscr{D} \subset \mathscr{D}(\mathbf{L})$. In this case the lattice has a specialized metric, which promotes its symmetry to a higher holohedry with respect to the holohedry of the individual. Nevertheless, the twin operation still belongs to $\mathscr{D}$.

1.2 Twinning by metric merohedry, or class IIB twins. Here, $\mathscr{H} \subseteq \mathscr{D} \subset \mathscr{D}(\mathbf{L})$, but now the twin operation belongs to $\mathscr{D}(\mathbf{L})$ and no longer to $\mathscr{D}$. Twinning by metric merohedry cannot occur for crystals belonging to the cubic or hexagonal crystal system and for trigonal crystals with an $h P$ lattice (see Fig. 3).

2 Twinning by pseudo-merohedry: $n=1$. It corresponds to an approximate metric merohedry: the lattice of the individual has a quasi-specialized metric. Twinning by pseudo-merohedry cannot occur for crystals belonging to the cubic or hexagonal lattice systems.

3.1 Twinning by reticular merohedry: $n>1, \omega=0$, $\mathscr{D}\left(\mathbf{L}_{\mathrm{T}}\right) \neq \mathscr{D}\left(\mathbf{L}_{\mathrm{I}}\right)$. The twin lattice is a sublattice of the individual lattice and the twin operation restores only a subset of the nodes of $\mathbf{L}_{\mathrm{I}}$. $\mathbf{L}_{\mathrm{T}}$ and $\mathbf{L}_{\mathrm{I}}$ correspond to different holohedries.

3.2 Twinning by reticular polyholohedry: $n>1$, $\omega=0, \mathscr{D}\left(\mathbf{L}_{\mathrm{T}}\right)=\mathscr{D}\left(\mathbf{L}_{\mathrm{I}}\right)$. This is a special case of reticular merohedry, where $\mathbf{L}_{\mathrm{T}}$ and $\mathbf{L}_{\mathrm{I}}$ belong to the same holohedry but some of their symmetry elements are not parallel. As a consequence, a subset of symmetry elements of $\mathbf{L}_{\mathrm{I}}$ is lost in $\mathbf{L}_{\mathrm{T}}$, and a corresponding subset reappears, in different orientation.

4.1 Twinning by reticular pseudo-merohedry: $n>1$, $\mathscr{D}\left(\mathbf{L}_{\mathrm{T}}\right) \neq \mathscr{D}\left(\mathbf{L}_{\mathrm{I}}\right)$. The various orientation states contain congruent sublattices in slightly different orientation. $\mathscr{D}\left(\mathbf{L}_{\mathrm{T}}\right)$ is the holohedry of these sublattices.

4.2 Twinning by reticular pseudo-polyholohedry: $n>1, \mathscr{D}\left(\mathbf{L}_{\mathrm{T}}\right)=\mathscr{D}\left(\mathbf{L}_{\mathrm{I}}\right)$. This is a special case of reticular pseudo-merohedry (see examples in $\mathrm{Ne}$ spolo and Ferraris, 2004).

Notice that Friedel's twins by merohedry have been split into the categories 1.1 and 1.2 , his twins by reticular merohedry into the categories 3.1 and 3.2, and his twins by reticular pseudo-merohedry into the categories 4.1 and 4.2. Friedel's definition of the obliquity as a parameter that fully characterises the pseudo-symmetry of a lattice is justified only in case of twofold twins.

\section{Application of coset analysis to twinning}

The interest in the coset decomposition of $\mathscr{K}^{*}$ with respect to $\mathscr{H}^{*}$ lies in the fact that each coset corresponds to a twin law. Detailed analyses of the coset decomposition of point groups to derive the possible twin laws are given in Flack (1987) and Hahn and Klapper (2003). Here we present a short summary with some examples.
We shall restrict our attention in the following to cases where the composite symmetry, $\mathscr{K}$ or $\mathscr{K}^{*}$, is a crystallographic point group.

\subsection{Twinning by merohedry}

In case of twinning by merohedry the point groups $\mathscr{H}_{i}$ of all the individuals of the twin have the same orientation, whence $\mathscr{H}^{*}=\mathscr{H}$. Notice that this is not always true for twinning by pseudo-merohedry. Let $N$ be the order of $\mathscr{H}$. Consider a first degree twin, with twin operation $y$ of order $n$. If $m$ is the least positive integer such that $g^{m}$ is a symmetry operation for the individual (in other words, $\mathscr{y}^{m} \in \mathscr{F}$ ), then $m$ is a factor of $n$ (possibly $n$ itself). The possible orientations of the symmetry element associated to $\mathscr{y}$ are restricted by the requirement that the group generated by $\mathscr{H}$ and $\mathscr{H}$ is a crystallographic point group $\mathscr{K}$ of order $N \times m$. The $N \times m$ operations obtained by multiplying each symmetry operation of $\mathscr{H}$ from the left by $\mathscr{y}^{j}$, where the integer $j$ varies in the range $1 \leq j \leq m$, are all different and belong to $\mathscr{K}$. Because $\mathscr{K}$ has order $N \times m$ they form the group $\mathscr{K}$; each of the $m$ sets obtained for a fixed value of $j$ is a left coset of $\mathscr{K}$ with respect to $\mathscr{H}$.

Let us write the point group by enumerating its elements: $\mathscr{H}=\left\{\mathscr{C}_{1}=1, \mathscr{h}_{2}, \mathscr{l}_{3}, \ldots, \mathscr{C}_{N}\right\}$, where 1 is the identity. ${ }^{l}$ To say that $g$ is an operation of order $n$ means that it is the generator of the Abelian group $\left\{\mathscr{y}, \mathscr{y}^{2}, \ldots, \mathscr{y}^{n}=1\right\}$. Let us suppose at first that the only power of $\mathscr{y}$ that belongs to $\mathscr{H}$ is $n$, where $\mathscr{g}^{n}=1$. The group $\mathscr{K}$ is then simply obtained as:

$$
\begin{aligned}
& \mathscr{g}\left\{1, \mathscr{h}_{2}, \mathscr{C}_{3}, \ldots \mathscr{h}_{N}\right\} \cup \mathscr{g}^{2}\left\{1, \mathscr{C}_{2}, \mathscr{h}_{3}, \ldots \mathscr{h}_{N}\right\} \\
& \cup \ldots \cup \mathscr{g}^{n}\left\{1, \mathscr{L}_{2}, \mathscr{h}_{3}, \ldots \mathscr{h}_{N}\right\} \\
& =\left(g, g h_{2}, g h_{3}, \ldots g h_{N}\right) \cup\left(g^{2}, g^{2} h_{2}, g^{2} h_{3}, \ldots g^{2} h_{N}\right) \\
& \cup \ldots \cup\left\{1, \mathscr{C}_{2}, \mathscr{C}_{3}, \ldots \mathscr{C}_{N}\right\}=\mathscr{g} \mathscr{H} \cup \mathscr{g}^{2} \mathscr{H} \cup \ldots \cup \mathscr{H} \text {, }
\end{aligned}
$$

where $\mathscr{g}^{j} \mathscr{H}$ denotes the coset obtained by multiplying $\mathscr{g}^{j}$ with each element of $\mathscr{H}$.

Example 1. Let us take $\mathscr{H}=3$ (the order of the group is 3 ) and let the twin operation be a rotation of order 2 about the same axis: $\mathscr{y}=2$. The group generated by $\mathscr{H}$ and $\mathscr{y}$ is a group $\mathscr{K}$ of order $2 \times 3=6$. Because in this case we have only two cosets, we shall make use of the dichromatic notation.

$$
\begin{aligned}
\mathscr{K}= & 2\left\{1,3,3^{-1}\right\} \cup 2^{2}\left\{1,3,3^{-1}\right\}=\left(2^{\prime}, 6^{-1 \prime}, 6^{\prime}\right) \\
& \cup\left\{1,3,3^{-1}\right\}=6^{\prime} .
\end{aligned}
$$

The resulting group contains the original group $\mathscr{H}=3$ and the coset $2 \mathscr{H}$; it is the dichromatic group $\mathscr{K}=6^{\prime}$ of order 6.

Twinning is by syngonic merohedry if the crystal has an $h P$ lattice (by reticular merohedry with $n=3$ if it has an $h R$ lattice).

Example 2. Let us take $\mathscr{H}=2 / m$ (the order of the group is 4) and $y=3$ (the order of the element is 3 ). The symmetry element associated to $\mathscr{y}$ can have two orientations

1 Following the common notation in group theory, the elements of a group are included in brackets, those of a coset in parentheses. 
with respect to the elements of $2 / m$, so that the result will be a crystallographic point group of order $4 \times 3=12$ : the three-fold axis can be either parallel to the two-fold axis of $2 / m$, or normal to it. In both cases the 3 -fold axis is taken parallel to [001].

3 || 2: We express $\mathscr{H}=2 / m$ in a $c$-unique setting, and multiply each element of $2 / m$ by $\mathscr{y}^{j}=3_{[001]}^{j}, 1 \leq j \leq 3$.

$$
\begin{aligned}
3_{[001]}\left\{1,2_{[001]}, \overline{1}, m_{[001]}\right\} \cup 3_{[001]}^{2}\left\{1,2_{[001]}, \overline{1}, m_{[001]}\right\} \\
\quad \cup 3_{[001]}^{3_{[001]}}\left\{1,2_{[001]}, \overline{1}_{[001]}\right\} \\
=\left(3_{[001],} 6^{-1}{ }_{[001]}, \overline{3}_{[001]}, \overline{6}^{-1}{ }_{[001]}\right) \\
\cup\left(3_{[001]}^{-1}, 6_{[001]}, \overline{3}^{-1}{ }_{[001]}, \overline{6}_{[001]}\right) \cup\left\{1,2_{[001]}, \overline{1}, m_{[001]}\right\}: \\
\mathscr{K}=6 / m
\end{aligned}
$$

$3 \perp$ 2: $\mathscr{H}=2 / m$ is expressed in a $b$-unique setting, and each of its elements is multiplied by $\mathscr{g}^{j}=3_{[001]}^{j}, 1 \leq j \leq 3$.

$3_{[001]}\left\{1,2_{[010]}, \overline{1}, m_{[010]}\right\} \cup 3_{[001]}^{2}\left\{1,2_{[010]}, \overline{1}, m_{[010]}\right\}$

$\cup 3_{[001]}^{3}\left\{1,2_{[010]}, \overline{1}, m_{[010]}\right\}=\left(3_{[001]}, 2_{[100]}, \overline{3}_{[001],} m_{[100]}\right)$

$\cup\left(3^{-1}{ }_{[001]}, 2_{[110]}, \overline{3}^{-1}{ }_{[001]}, m_{[110]}\right) \cup\left\{1,2_{[010]}, \overline{1}, m_{[010]}\right\}:$

$\mathscr{K}=\overline{3} m$

In both cases, $\mathscr{K}$ corresponds to a trichromatic point group: for the notation of polychromatic groups see $\mathrm{Ne}$ spolo (2004). Twinning is by metric merohedry, i.e. 3 || 2 is possible only if the lattice is of type $h P$ with its 6-fold axis parallel to the monoclinic axis; $3 \perp 2$ is possible only if the lattice is of type $h P$ or $h R$ with the monoclinic axis parallel to one of the 2 -fold axes of the lattice.

So far we have analyzed only first-degree twins. The derivation of higher-degree twins follows the same route. The group of an $m$-th degree twin $\mathscr{K}^{m}$ is obtained from the group of the individual $\mathscr{H}$ in $m$ steps through the intermediate groups $\mathscr{K}^{1}, \mathscr{K}^{2} \ldots \mathscr{K}^{m}$.

Example 3. Let us consider a second-degree twin, i.e. a twin in which two independent twin elements $\mathscr{H}_{1}$ and $\mathscr{g}_{2}$ operate. Let $\mathscr{H}=222, \mathscr{H}_{1}=3_{[111]}$ and $\mathscr{g}_{2}=4_{[001]}$. We obtain $\mathscr{K}^{2}$ in two steps, first by applying $\mathscr{H}_{1}$ (order 3 ) to $\mathscr{H}$ and obtaining the intermediate group $\mathscr{K}^{1}=23$ (order

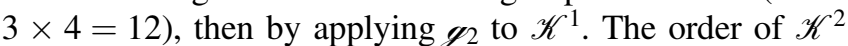
is $2 \times 12=24$ because $\mathscr{y}_{2}^{2} \in \mathscr{H} . \mathscr{K}^{2}=432$ corresponds to a hexachromatic crystallographic point group.

To obtain the twin laws when $\mathscr{K}$ is known, we apply the opposite process, namely the coset decomposition of $\mathscr{K}$ in terms of $\mathscr{H}$. Each coset represents a possible twin law, and each element of the coset is a possible twin operation. From the practical viewpoint, $\mathscr{K}$ is supposed to be known: it is revealed, for example, by the diffraction pattern. $\mathscr{H}$ is not necessarily known in advance. Several hypotheses can be made on $\mathscr{H}_{3}$ the corresponding twin laws derived and tested against the experimental data.

Example 4. Let us suppose that the diffraction pattern of a crystal is consistent with a tetragonal lattice geometry; we therefore take $\mathscr{D}\left(\mathbf{L}_{\mathrm{I}}\right)=4 / \mathrm{mmm}$. Suppose that the symmetry of the intensities is $\mathscr{K}_{1}=4 / m$ but that the structure cannot be refined, neither assuming a single crystal with $\mathscr{H}=4, \overline{4}$ or $4 / m$, nor a twinned crystal with $\mathscr{H}=4$ or $\overline{4}$.
Therefore we suspect second-degree twinning with $\mathscr{K}=4 / \mathrm{mmm}$. Both for $\mathscr{H}=4$ and for $\mathscr{H}=\overline{4}$, the coset decomposition can be obtained as follows:

$$
\begin{aligned}
\mathscr{K}_{1}= & \mathscr{H} \cup \overline{1} \mathscr{H} ; \\
\mathscr{K}= & \mathscr{K}_{1} \cup 2_{[100]} \mathscr{K}_{1}=\mathscr{H} \cup \overline{1} \mathscr{H} \cup 2_{[100]} \mathscr{H} \\
& \cup m_{[100]} \mathscr{H} .
\end{aligned}
$$

This decomposition of $\mathscr{K}=4 / \mathrm{mmm}$ into four cosets (including the subgroup $\mathscr{H}=4$ or $\overline{4}$ ) describes a second-degree twin, which may contain four different orientation states. Each of the four twin laws corresponds to four twin operations, which may alternatively be used to describe the twin.

Twinning is by syngonic merohedry.

\subsection{Twinning by reticular (pseudo) merohedry/ polyholohedry}

For $n>1, \mathscr{D}\left(\mathbf{L}_{\mathrm{T}}\right)$ is no longer limited to a supergroup of $\mathscr{H}$. Let us enumerate the different orientation states in a twin by an index $1,2, . . j . . v . \mathbf{L}_{j}$ may have some of its symmetry elements oriented differently from those of $\mathbf{L}_{k \neq j}$ : these elements are not retained in $\mathbf{L}_{\mathrm{T}}$. On the contrary, those elements that are common to all $\mathbf{L}_{j}(j=1, v)$ are symmetry elements of $\mathbf{L}_{\mathrm{T}}$ too. They define the intersection group $\mathscr{D}^{*}$ of $\mathscr{D}\left(\mathbf{L}_{1}\right), \mathscr{D}\left(\mathbf{L}_{2}\right), \ldots \mathscr{D}\left(\mathbf{L}_{v}\right) . \mathscr{D}\left(\mathbf{L}_{\mathrm{T}}\right)$ is finally a supergroup of $\mathscr{D}^{*}$, which may not contain a subgroup of type $\mathscr{D}\left(\mathbf{L}_{1}\right)$. If $\mathscr{D}\left(\mathbf{L}_{\mathrm{T}}\right)$ and $\mathscr{D}\left(\mathbf{L}_{1}\right)$ are of different type, twinning is by reticular merohedry; if they are of the same type, twinning is by reticular polyholohedry.

Example 1. The dovetail twin of gypsum, $\mathrm{CaSO}_{4} \cdot 2 \mathrm{H}_{2} \mathrm{O}$. The space group of the individual is $A 2 / a, a=5.679 \AA$, $b=15.202 \AA, c=6.287 \AA, \beta=114.17^{\circ}$ (Pedersen and Semmingsen, 1982, converted from $I 2 / a$ to $A 2 / a$ ), and thus $\mathscr{H}=12 / \mathrm{m} 1$; the twin operation is the reflection in the mirror plane $m_{(100)}$. We choose a new orthorhombic basis system with $\boldsymbol{b}^{\prime}=\boldsymbol{b}, \boldsymbol{c}^{\prime}=\boldsymbol{c}$ and $\boldsymbol{a}^{\prime}$ perpendicular to the twin plane. In this coordinate system, the twin plane can be designated $m_{[100]}$ (see Fig. 5).

The alternative twin operations are given in Table 7, expressed in the orthorhombic coordinate system.

The twin point group $\mathscr{K}$ corresponds to the dichromatic group:

$$
\begin{aligned}
& \frac{2^{\prime}}{m^{\prime}} \frac{2}{m} \frac{2^{\prime}}{m^{\prime}} \\
& =\left\{1,2_{[100]}^{\prime}, 2_{[010]}, 2_{[001]}^{\prime}, \overline{1}, m_{(100)}^{\prime}, m_{(010)}, m_{(001)}^{\prime}\right\} .
\end{aligned}
$$

The monoclinic direction [301] is pseudo-normal to the twin mirror plane $m_{(100)}$. The cell of $\mathbf{L}_{T}$ is defined by these two elements. Twin index is 3 and twin obliquity is

Table 7. The twin law of gypsum expressed in orthorhombic coordinates.

\begin{tabular}{ll}
\hline $\begin{array}{l}\text { Crystal symmetry } \\
\mathscr{H}=12 / m 1\end{array}$ & $\begin{array}{l}\text { Twin law }=\operatorname{coset} \mathscr{g} \mathscr{H} \\
\text { of alternative twin operations }\end{array}$ \\
\hline 1 & $m_{[100]} 1=m_{[100]}$ \\
$2_{[010]}$ & $m_{[100]} 2_{[010]}=m_{[001]}$ \\
$\overline{1}$ & $m_{[100]} \overline{1}=2_{[100]}$ \\
$m_{[010]}$ & $m_{[100]} m_{[010]}=2_{[001]}$ \\
\hline
\end{tabular}



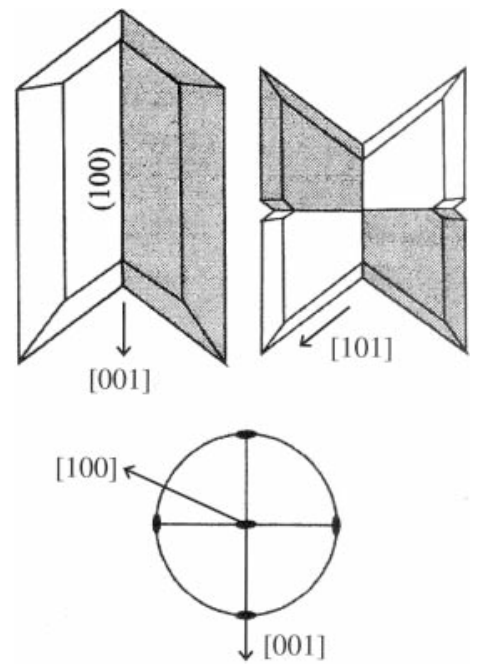

Fig. 5. The dovetail twin of gypsum. On the left the most frequent form consisting of two individuals; on the right the rare form with four individuals, displaying the orthorhombic composite symmetry. The two orientation states are distinguished by shading (after HahnKlapper (2003) with the permission of the IUCr).

$2.54^{\circ}$. Dovetail twinning in gypsum is by reticular pseudomerohedry.

$\mathbf{L}_{\mathrm{T}}$ is pseudo-orthorhombic, and its (pseudo-)symmetry elements have the following orientation with respect to the corresponding lattice planes/directions of $\mathbf{L}_{1}$ :

$$
\begin{array}{lll}
{[100]_{\mathrm{T}} \|[301]_{1} ;} & {[010]_{\mathrm{T}} \|[010]_{1} ;} & {[001]_{\mathrm{T}} \|[001]_{1},} \\
(100)_{\mathrm{T}} \|(100)_{1} ; & (010)_{\mathrm{T}} \|(010)_{1} ; & (001)_{\mathrm{T}} \|(103)_{1} .
\end{array}
$$

Example 2. We consider a twinned crystal with general metric and point group $\mathscr{H}=222$ and assume that the twin operation $k$ is a 2 -fold rotation with axis [uv0], where $u$ and $v$ are coprime integers different from zero. The axis of $k$ is therefore normal to [001], $\mathscr{H}^{*}=\left\{1,2_{[001]}\right\}$ and $\mathscr{K}^{*}=222$. $\mathscr{H}$ and $\mathscr{K}^{*}$ are point groups of the same type having different orientations. This type of twinning is called twinning by reticular polyholohedry if the plane normal to $[u v 0]$ is a lattice plane, and twinning by reticular pseudo-polyholohedry otherwise. In the first case $\mathbf{L}_{T}$ is orthorhombic, in the second case it is monoclinic pseudo-orthorhombic.

If now we take three individuals instead of two, related by a 3 -fold axis parallel to [001], the group $\mathscr{K}^{*}$ becomes:

$$
\begin{aligned}
\mathscr{K}= & 3_{[001]}\left\{1,2_{[001]}\right\} \cup 3_{[001]}^{2}\left\{1,2_{[001]}\right\} \\
& \cup 3_{[001]}^{3}\left\{1,2_{[001]}\right\} \\
= & \left\{3_{[001]}, 6_{[001]}^{-1}, 3^{-1}{ }_{[001]}, 6_{[001]}, 1,2_{[001]}\right\}=6 .
\end{aligned}
$$

For the crystal association to be a twin, it must have a (pseudo)hexagonal $\mathbf{L}_{\mathrm{T}}$, which exists if the ratio b/a for the individual lattices is not far from a rational multiple of $3^{1 / 2}$. Twinning is by reticular (pseudo) merohedry.

Starting from $\mathscr{K}^{*}$, its coset decomposition is performed in the same way as shown for the case of (pseudo) merohedry. However, the group in terms of which the decomposition is performed is $\mathscr{H}^{*}$, not $\mathscr{H}$. The point group of the individual is thus in general a supergroup of that used in the coset decomposition: it may be of the same type as $\mathscr{K}^{*}$ - reticular (pseudo) polyholohedry - or not - reticular (pseudo) merohedry.

\section{The Coincidence-Site Lattice (CSL)}

A lattice is brought into self-coincidence by any symmetry operation of the corresponding holohedry. A rotation about a lattice point that does not belong to the symmetry operations of the holohedry, in general, does not restore any other node; for well-defined rotation angles and axes, however, a partial self-coincidence is obtained. The restored nodes then form a one-, two- or three-dimensional lattice. In the latter case one speaks of a coincidence-site lattice (CSL), a term introduced by Brandon, Ralph, Ranganathan and Wald (1964). Already 15 years earlier, Kronberg and Wilson (1949) had observed that neighbouring grains in copper that had undergone secondary recrystallization often were related by a $22^{\circ}$ or $38^{\circ}$ rotation about a [111] axis, which give rise to coincidence of every seventh lattice node in each (111) plane in case of the $38^{\circ}$ rotation or in every third (111) plane in case of the $22^{\circ}$ rotation (cf. Table 11 below, where the numbers given in column $\Sigma_{h R}$ hold also in the special case of rotations about a 3-fold axis of cubic lattices). Brandon (1966) generalized these ideas to the CSL model of high-angle grain boundaries. He assumed that two neighbouring grains in coincidence orientation have certain atom positions at the grain boundary in common. Later observations by highresolution transmission electron microscopy as well as computer simulations showed (see e.g. the review by Fischmeister, 1985) that, in general, the two grains are not related by a rotation about a common atom position but by such a rotation combined with a translation. Two grains related in such a way still have symmetry translations in common, forming what more appropriately would be called a 'coincidence vector lattice'.

The CSL model of grain boundaries in polycrystalline materials therefore claims that neighbouring grains often have a large proportion of symmetry translations (i.e. lattice vectors) in common. This proportion can be characterized as follows. Let $V$ be the volume of a smallest (i.e. primitive) cell spanned by the lattice vectors of one of the grains and $V_{C}$ the volume of a smallest cell spanned by the lattice vectors that the two grains have in common. Then the ratio $\Sigma=V_{C} / V$ is called the multiplicity (of the CSL). We speak of a coincidence site lattice if $V_{C}$ is finite, in which case $\Sigma$ is an integer $\geq 1$. For small values of $\Sigma$ the relative orientation of the lattices of the two grains can often be described by a $180^{\circ}$ rotation. The axis of this rotation is then a 2-fold symmetry axis of the CSL; the multiplicity coincides with the twin index and can be computed using Table 4.

The twin lattice $\mathbf{L}_{T}$ described above is a CSL of low multiplicity, and the interest of the CSL theory for the study of twins is thus evident. However, the CSL model has wider applications. For example, also the lattice generated by the lattice vectors of both grains is of physical interest. Bollmann (1967b) called it the complete patternshift lattice or "displacement shift complete lattice" (DSC lattice or DSCL); its vectors are the geometrically possible Burgers vectors of perfect grain boundary dislocations. The volume $V_{D}$ of a smallest cell of the DSCL is $V_{D}=V / \Sigma$.

Several different approaches to derive CSL lattices have been proposed, like the 0-lattice theory (Bollmann, 
1967a, b; 1970), consideration of C-lattices (Fortes, 1977), or the application of the elementary divisor theorem (Grimmer, 1976; Fortes 1983a, b). Closely related to the determination of CSLs are also the theory of image sets (Buerger, 1961) and the compound-tessellation theory (Coxeter, 1973, 1989; Takeda and Donnay, 1965).

Example. Thin $\mathrm{MgO}$ substrates for use in transmission electron microscopy were prepared by chemical thinning of cleaved $\{001\}$ sections (Mykura, Bansal and Lewis, 1980). Smoke particles obtained by burning magnesium metal in air were collected on these substrates. The smoke particles were approximately cube-shaped with their 4-fold axes parallel to the edges of the cube. It follows that the substrate and each of the smoke particles on it have two 4-fold axes in the contact plane and one normal to it. The relative orientation of a smoke particle and the substrate can therefore be described by a rotation about the normal to the contact plane with angle $\theta$ in the range $0^{\circ} \leq \theta^{\circ} \leq 45^{\circ}$. Fig. 6 shows the distribution of $\theta$ for the 344 particles collected.

The histogram has four marked peaks. The highest corresponds to particles whose symmetry axes are parallel to those of the substrate. Consider the lattice vectors of the smoke particle and of the substrate that lie in the contact plane. We shall show later that the fraction $1 / \sigma$ of such vectors that agree in the substrate and a smoke particle can take the values given by $\sigma=1,5,13,17,25, \ldots$ The corresponding values of $\theta$ are $0^{\circ}, 36.87^{\circ}, 22.62^{\circ}, 28.07^{\circ}$, $16.26^{\circ}, \ldots$ Notice that the five highest peaks in Fig. 6 correspond exactly to these $\theta$ values and that the height of the peaks decreases with increasing value of $\sigma$ with only one exception. We conclude that when the $\mathrm{MgO}$ particles approach the substrate they turn one of their 4-fold axes normal to the surface of the substrate and can rotate a few degrees about this axis to approach an energetically favourable value of $\theta$. Energetically favourable are the values that correspond to the lowest values of $\sigma$ according to the results of Mykura, Bansal and Lewis (1980). For rotations about [001] the fraction of lattice vectors that are

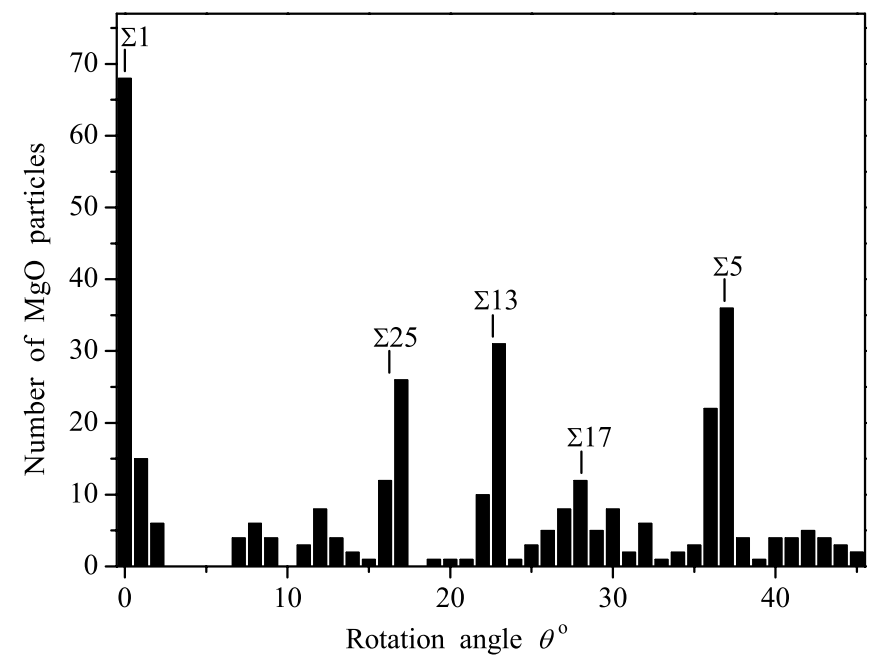

Fig. 6. Histogram of the number of $\mathrm{MgO}$ cubes in $1^{\circ}$ intervals of the rotation angle $\theta$ about $\langle 001\rangle$ for $\mathrm{MgO}$ smoke particles on a $\{001\}$ surface of $\mathrm{MgO}$. coincidence vectors has the same value $1 / \sigma$ in each $(001)$ plane. The planar fraction $1 / \sigma$ therefore agrees with the spatial fraction $1 / \Sigma$, i.e. $\sigma=\Sigma$.

\subsection{The case of cubic lattices}

In texts on coincidence site lattices, the relative orientation of two congruent lattices is usually called their misorientation and described by a rotation relating conventional bases of the two lattices. In the case of cubic lattices, rotations that give rise to a CSL can be characterized by a quadruple of integers $(m, U, V, W)$ without common divisor, where $[U V W]$ is the axis and

$$
\theta=2 \arctan \left(\frac{1}{m} \sqrt{U^{2}+V^{2}+W^{2}}\right)
$$

the angle of the rotation. The angle $\theta$ has the same sign as $m$. The rotation with angle $|\theta|$ around $[U V W]$ is anticlockwise (right-handed) for $m>0$ and clockwise for $m<0$. The quadruple $(-m, U, V, W)$ describes the inverse rotation to $(m, U, V, W)$, but the rotations for $\pm(m, U, V, W)$ are identical.

Due to the rotational symmetry 432 of the cubic lattice, which contains 24 group elements, and the interchangeability of the two congruent lattices, there are in general $2 \times 24 \times 24=1152$ rotations expressing the same misorientation; they will be called (cubically) equivalent. For certain misorientations, several of these rotations are identical. The actual number of distinguishable equivalent rotations is always a multiple of 24 and a divisor of 1152, and

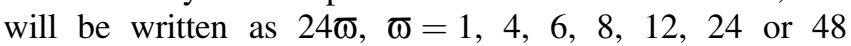
(Grimmer, 1973). The number of different equivalent quadruples is $48 \sigma$ because $\pm(m, U, V, W)$ describe the same rotation.

Among equivalent rotations with the same rotation angle there is at least one with axis in the standard spherical triangle: $U \geq V \geq W \geq 0$. Such a rotation with minimum angle, called the reduced rotation, and the corresponding quadruple $(m, U, V, W)$ with non-negative components is typically chosen to represent the misorientation. It satisfies $m \geq U \geq V \geq W \geq 0$. The first inequality follows for cubic lattices from the minimum angle requirement (Grimmer, 1973).

Most sets of equivalent rotations for CSLs with a low value of $\Sigma$ contain also $180^{\circ}$ rotations (The first exception occurs for one of the sets with $\Sigma=39$ ). The misorientation described by $\pm(m, U, V, W)$, where $m, U, V$ and $W$ are integers without common divisor, gives rise to a CSL with $\Sigma$ equal to the largest odd factor of $m^{2}+U^{2}+V^{2}+W^{2}$. This is true for all three types of cubic lattices, $c P, c I$ and $c F$. In the special case of $180^{\circ}$ rotations, we have $m=0$. The sum of the four squares therefore reduces to $S=U^{2}+V^{2}+W^{2}$, which contains at most one factor 2 . It follows that $\Sigma=S$ if $S$ is odd and $\Sigma=S / 2$ if $S$ is even, which coincides with the formula for the twin index $n$ given earlier.

Consider the special case of rotations with axis [001]. Then $U=V=0$. To determine the five lowest values of $\Sigma$ that are possible in this case, we may assume $m \geq W \geq 0$ and obtain the result given in Table 8 . 
Table 8. Determination of the lowest multiplicities $\Sigma$ that are possible for rotations about [001] of a cubic lattice and of the corresponding rotation angles $\theta$.

\begin{tabular}{cccccc}
\hline$m$ & $W$ & $S=m^{2}+W^{2}$ & \multicolumn{1}{c}{$\Sigma$} & $\tan (\theta / 2)=W / m$ & $\theta\left(^{\circ}\right)$ \\
\hline 1 & 0 & 1 & $\mathbf{1}$ & 0 & 0 \\
1 & 1 & 2 & $\mathbf{1}$ & 1 & 90 \\
2 & 1 & 5 & $\mathbf{5}$ & $1 / 2$ & 53.13 \\
3 & 1 & 10 & $\mathbf{5}$ & $1 / 3$ & 36.87 \\
3 & 2 & 13 & $\mathbf{1 3}$ & $2 / 3$ & 67.38 \\
4 & 1 & 17 & $\mathbf{1 7}$ & $1 / 4$ & 28.07 \\
4 & 3 & 25 & $\mathbf{2 5}$ & $3 / 4$ & 73.74 \\
5 & 1 & 26 & $\mathbf{1 3}$ & $1 / 5$ & 22.62 \\
5 & 2 & 29 & 29 & & \\
5 & 3 & 34 & $\mathbf{1 7}$ & $3 / 5$ & 61.93 \\
5 & 4 & 41 & 41 & & \\
6 & 1 & 37 & 37 & & \\
6 & 5 & 61 & 61 & & \\
7 & 1 & 50 & $\mathbf{2 5}$ & $1 / 7$ & \\
& & $>50$ & $>25$ & & \\
\hline
\end{tabular}

The positive integer $W$ in Table 8 determines (together with $m$ ) the rotation angle $\theta$; the rotation axis is always [001]. Notice that the two values of $\theta$ that appear for a given value of $\Sigma$ add up to $90^{\circ}$, so that there is always a solution $\theta \leq 45^{\circ}$. The five highest peaks in Fig. 6 correspond to the $\theta$ values $<45^{\circ}$ for the five values of $\Sigma \leq 25$, as mentioned earlier. The example $m=3, W=1$ is illustrated in Fig. 7.

In contrast to twins that formed during nucleation, the aggregate of $\mathrm{MgO}$ substrate and smoke particles formed when the smoke crystals fell onto the substrate. Such aggregates with preferred misorientation often corresponding to large values of $\Sigma$, and formed by coalescence of macroscopic crystals were called plesiotwins by Nespolo, Ferraris, Takeda and Takéuchi (1999).

Important new results on CSL grain boundary networks in cubic crystals have recently been obtained by Reed, Minich, Rudd and Kumar (2004).

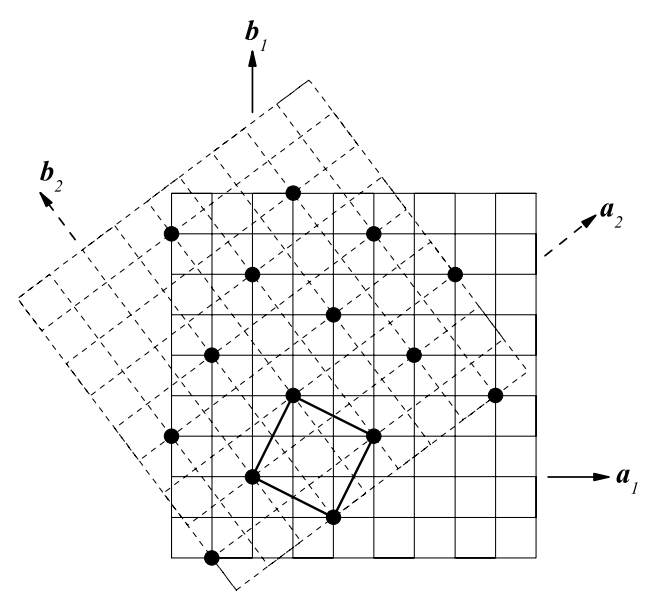

Fig. 7. If a square net is rotated by $\theta=36.87^{\circ}$ about one of its nodes, the initial and rotated net have $1 / 5$ of their nodes in common.

\subsection{Coincidence misorientations of tetragonal lattices}

The misorientation of two congruent tetragonal lattices can be described by a rotation relating conventional bases of the two lattices. Some rotations lead to CSLs in every tetragonal lattice. These are the common rotations, for which $\Sigma$ does not depend on the axial ratio $c / a$. If $c^{2} / a^{2}$ is a rational number, then there exist additional specific rotations. ${ }^{2}$ Common and specific rotations can be characterized by quadruples of integers $(m, U, V, W)$ without common divisor, where $[U V W]$ is the axis and

$$
\theta=2 \arctan \left(\frac{1}{m} \sqrt{\frac{a^{2}\left(U^{2}+V^{2}\right)+c^{2} W^{2}}{c^{2}}}\right)
$$

the angle of the rotation. The same conventions on the sign of $\theta$ and the sense of the rotation hold as in the cubic case. The quadruple $(-m, U, V, W)$ describes the inverse rotation to $(m, U, V, W)$, but the rotations for $\pm(m, U, V, W)$ are identical. Due to the rotational symmetry 422 of the tetragonal lattice, which contains 8 group elements, and the interchangeability of the two congruent lattices, there are in general $2 \times 8 \times 8=128$ rotations expressing the same misorientation; they will be called equivalent. For certain misorientations, several of these rotations are identical. The actual number of distinguishable equivalent rotations is always a multiple of 8 and a divisor of 128 , and will be written as $8 \varpi, \varpi=1,2,4,8$ or 16 (Grimmer, 1980). The number of different equivalent quadruples is $16 \Phi$ because $\pm(m, U, V, W)$ describe the same rotation. A quadruple $(m, U, V, W)$ describes a common rotation if either $U=V=0$ or $m=W=0$. In the first case we have a rotation with axis $\| c$, in the second a $180^{\circ}$ rotation with axis $\perp c$.

Among equivalent rotations with the same rotation angle there is at least one with axis in the standard spherical triangle: $U \geq V \geq 0, W \geq 0$. Such a rotation with minimum angle, called the reduced rotation, and the corresponding quadruple $(m, U, V, W)$ with non-negative components is typically chosen to represent the misorientation (Singh and King, 1993).

For a given value of $c / a$ and a given misorientation the multiplicity will generally depend on the centring type of the lattice. It will be called $\Sigma_{P}$ in the case of $t P$ lattices and $\Sigma_{I}$ for $t I$ lattices. Grimmer (1993) showed that $\Sigma_{I}=\Sigma_{P} / 2, \Sigma_{P}$ or $2 \Sigma_{P}$. For common misorientations we have $\Sigma_{P}=\Sigma_{I}=\Sigma$. Those with $\Sigma \leq 50$ are listed in Table 9. In all the cases listed in the table the Bravais types of the CSL and the DSCL coincide with the Bravais type of the crystal lattice. Common misorientations in tetragonal lattices therefore give rise to twinning by reticular polyholohedry.

The specific rotations with $\Sigma \leq 5$ were determined by Grimmer (2003) for $t P$ and $t I$ lattices. Those with $\Sigma \leq 4$ are listed in Table 10. These misorientations correspond to twinning by reticular merohedry if the axial ratio $c / a$ of the tetragonal lattice has the indicated value, where $(c / a)^{2}$ has the rational value $\mu / v$; the misorientation corresponds

2 Some authors call the common rotations exact (e.g. Singh and King, 1993), the specific rotations approximate or constrained. 
Table 9. The common misorientations with $\Sigma \leq 50$. Each misorientation is given by its representative quadruple $(m, U, V, W)$ and the corresponding minimum angle rotation $\theta$. Also the axes $[u v w]$ of equivalent $180^{\circ}$ rotations in the standard spherical triangle are listed; the axis $[u v w]$ and the plane $(h k l)$ perpendicular to it, called twin mirror plane, have the same indices.

\begin{tabular}{|c|c|c|c|c|c|c|c|c|c|c|c|c|}
\hline \multirow[t]{3}{*}{$\Sigma$} & \multirow[t]{3}{*}{$\varpi$} & \multicolumn{5}{|c|}{ Representative } & \multicolumn{6}{|c|}{ Twin Mirror Planes } \\
\hline & & $\theta^{\circ}$ & $m$ & $U$ & $V$ & $W$ & $h$ & $k$ & $l$ & $h$ & $k$ & $l$ \\
\hline & & & & & & & $u$ & $v$ & $w$ & $u$ & $v$ & $w$ \\
\hline 5 & 2 & 36.87 & 3 & 0 & 0 & 1 & 3 & 1 & 0 & 2 & 1 & 0 \\
\hline 13 & 2 & 22.62 & 5 & 0 & 0 & 1 & 5 & 1 & 0 & 3 & 2 & 0 \\
\hline 17 & 2 & 28.07 & 4 & 0 & 0 & 1 & 4 & 1 & 0 & 5 & 3 & 0 \\
\hline 25 & 2 & 16.26 & 7 & 0 & 0 & 1 & 7 & 1 & 0 & 4 & 3 & 0 \\
\hline 29 & 2 & 43.60 & 5 & 0 & 0 & 2 & 5 & 2 & 0 & 7 & 3 & 0 \\
\hline 37 & 2 & 18.92 & 6 & 0 & 0 & 1 & 6 & 1 & 0 & 7 & 5 & 0 \\
\hline 41 & 2 & 12.68 & 9 & 0 & 0 & 1 & 9 & 1 & 0 & 5 & 4 & 0 \\
\hline
\end{tabular}

to twinning by reticular pseudo-merohedry if the axial ratio slightly deviates from the indicated value. The primitive tetragonal lattice becomes a primitive cubic lattice if $c / a=1$. Except in the last two columns we deal with it as if it had only tetragonal symmetry in order to see what happens if the axial ratio slightly deviates from 1 . If the axial ratio is exactly 1 then the specific rotation with $\Sigma=1$ becomes a symmetry rotation of the cubic lattice and the two specific rotations with $\Sigma=3$ describe the same misorientation of two congruent cubic lattices, namely the one occurring for the spinel law of twinning. The last two columns show that the CSL may have a higher (cubic), a different (hexagonal), the same (tetragonal) or a lower (orthorhombic) holohedry than the tetragonal lattice of the individual.

If two lattices have primitive bases $\mathbf{e}_{i}$ and $\mathbf{f}_{i}, i=1,2$, 3 , satisfying $\mathbf{e}_{i} \cdot \mathbf{f}_{j}=k \delta_{i j}$, where $k$ is a constant of dimension length squared, then we shall call these two lattices pseudoreciprocal. When $k=V^{2 / 3}, V$ being the volume of the unit cell, the pseudoreciprocal lattice coincides with Bravais' polar lattice (Bravais, 1850). Examples: the cubic lattices $c I$ and $c F$ are pseudoreciprocal; $c P$ is pseudoreci-

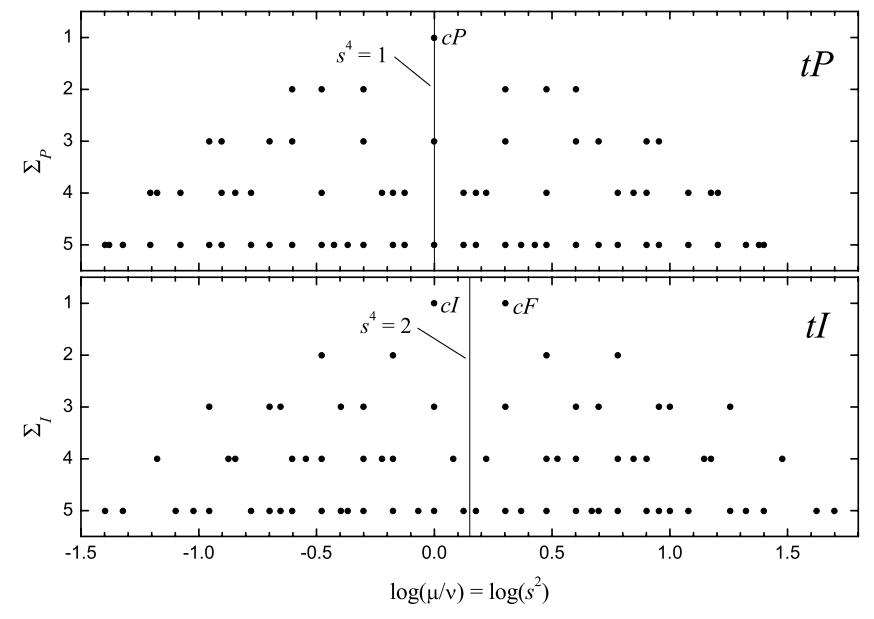

Fig. 8. Specific misorientations of primitive $(t P)$ and body-centred $(t I)$ tetragonal lattices with multiplicity (twin index) $\Sigma \leq 5$ as a function of the axial ratio $s$. Notice that the three types of cubic lattices appear as special cases of the tetragonal types: $c P$ as a special case of $t P, c I$ and $c F$ as special cases of $t I$. procal to itself. The $t P$ lattice with $\mu=m, v=n$ is pseudoreciprocal to the $t P$ lattice with $\mu=n, v=m$; the $t I$ lattice with $\mu=m, v=n$ ( $m$ odd) is pseudoreciprocal to the $t I$ lattice with $\mu=2 n, v=m$ [i.e. the $t F$ lattice with $\mu=n, v=m]$ (see Fig. 8).

Notice that in Table 10 to each specific rotation there exists a specific rotation of the pseudoreciprocal lattice with the same values of $\Sigma_{P}, \varpi$ and $i$. The two representative rotations have the same angle $\theta$ (because $i$ and $\Sigma_{P}$ are the same). The twin mirror plane columns show that the triples $h k l$ and $u v w$ are interchanged for pseudoreciprocal lattices. The resulting CSL is pseudoreciprocal to the DSCL of the pseudoreciprocal lattice and vice versa. ${ }^{3}$ This is a manifestation of the reciprocity relation proved by Grimmer (1974).

\subsection{Coincidence misorientations of hexagonal and rhombohedral lattices}

The situation for primitive hexagonal $(h P)$ lattices and rhombohedral $(h R)$ lattices is similar to that for tetragonal lattices, as has been shown in detail in Grimmer and Kunze (2004). We shall not treat these cases here in detail but only point out some aspects of particular interest in connection with twinning. Also for $h P$ and $h R$ lattices common and specific rotations have to be distinguished. Unlike for $t P$ and $t I$ lattices, which have the same holohedry $4 / \mathrm{mmm}$, the holohedry $\overline{3} m$ of $h R$ has only half as many elements as the holohedry $6 / \mathrm{mmm}$ of $h P$. The maximum number of equivalent rotations is therefore four times smaller for $h R$ than for $h P$, and a set of rotations that describe the same misorientation of two congruent $h P$ lattices may split into up to four sets, each set describing a different misorientation of two congruent $h R$ lattices. The simple case of common rotations is described in Table 11 .

Whereas two rotations about the principal symmetry axis of a $h P$ lattice with angles $\theta$ and $60^{\circ}-\theta$ describe the same misorientation, this is no longer true for $h R$ lattices: each set of common rotations that describe the same misorientation of two congruent $h P$ lattices splits into two sets, each set describing a different misorientation of two congruent $h R$ lattices. $\Sigma_{h P}$ of a common rotation is never a multiple of 3 ; one of the corresponding values of $\Sigma_{h R}$ equals $\Sigma_{h P}$, the other is 3 times larger. Consider in particular the set of all symmetry rotations of a $h P$ lattice: half of these rotations are symmetry rotations of $h R$, the other half describe the misorientation of the $\Sigma=3$ twin by reticular merohedry, which is frequently observed in many crystals with a $h R$ lattice. If the individual lattice is of type $h P$ then the CSL has the same type (i.e. we have reticular polyholohedry); if the individual lattice is of type $h R$ then the CSL has the same type if $\Sigma_{h R}$ is not a multiple of 3 (reticular polyholohedry), whereas the CSL has type $h P$ if $\Sigma_{h R}$ is a multiple of 3 .

The specific rotations with $\Sigma \leq 5$ for $h P$ and $h R$ lattices are illustrated in Fig. 9.

\footnotetext{
${ }^{3}$ Pseudoreciprocal lattices have the same Bravais type with two exceptions: $c I \leftrightarrow c F$ and $o I \leftrightarrow o F$.
} 
Table 10. The specific misorientations with $\Sigma \leq 4$ for $t P$ lattices. Each misorientation is given by its representative quadruple $(m, U, V, W)$; the corresponding minimum angle rotation $\theta$ satisfies $\cos \theta=i /\left(2 \Sigma_{P}\right)$. Also the axes [uvw] of equivalent $180^{\circ}$ rotations in the standard spherical triangle are listed. The plane normal to $[u v w]$ is called the twin mirror plane $(h k l)$. The last two columns give the Bravais type of the CSL and DSCL, respectively.

\begin{tabular}{|c|c|c|c|c|c|c|c|c|c|c|c|c|c|c|c|c|c|c|c|c|c|c|c|}
\hline \multirow[t]{2}{*}{$\Sigma_{P}$} & \multirow[t]{2}{*}{$\mu$} & \multirow[t]{2}{*}{$v$} & \multirow[t]{2}{*}{$\varpi$} & \multirow[t]{2}{*}{$c / a$} & \multicolumn{4}{|c|}{ Representative } & \multicolumn{7}{|c|}{$\begin{array}{l}\text { Twin Mirror } \\
\text { Plane } 1\end{array}$} & \multicolumn{6}{|c|}{$\begin{array}{l}\text { Twin Mirror } \\
\text { Plane } 2\end{array}$} & \multirow[t]{2}{*}{ CSL } & \multirow[t]{2}{*}{ DSCL } \\
\hline & & & & & $m$ & $U$ & $V$ & $W$ & $i$ & $h$ & $k$ & $l$ & $u$ & $v$ & $w$ & $h$ & $k$ & $l$ & $u$ & $v$ & $w$ & & \\
\hline 1 & 1 & 1 & 2 & 1. & 1 & 1 & 0 & 0 & 0 & 1 & 0 & 1 & 1 & 0 & 1 & & & & & & & $c P$ & $c P$ \\
\hline \multirow[t]{6}{*}{2} & 1 & 4 & 2 & 0.5 & 2 & 1 & 0 & 0 & 0 & 2 & 0 & 1 & 1 & 0 & 2 & & & & & & & $c P$ & $t P$ \\
\hline & 1 & 3 & 4 & 0.5774 & 3 & 1 & 0 & 0 & 2 & 1 & 0 & 1 & 1 & 0 & 3 & 3 & 0 & 1 & 1 & 0 & 1 & $h P$ & $h P$ \\
\hline & 1 & 2 & 2 & 0,7071 & 2 & 1 & 1 & 0 & 0 & 1 & 1 & 1 & 1 & 1 & 2 & & & & & & & $c I$ & $c P$ \\
\hline & 2 & 1 & 2 & 1.4142 & 1 & 1 & 1 & 0 & 0 & 1 & 1 & 2 & 1 & 1 & 1 & & & & & & & $c P$ & $c F$ \\
\hline & 3 & 1 & 4 & 1.7321 & 1 & 1 & 0 & 0 & 2 & 1 & 0 & 3 & 1 & 0 & 1 & 1 & 0 & 1 & 3 & 0 & 1 & $h P$ & $h P$ \\
\hline & 4 & 1 & 2 & 2. & 1 & 2 & 0 & 0 & 0 & 1 & 0 & 2 & 2 & 0 & 1 & & & & & & & $t P$ & $c P$ \\
\hline \multirow[t]{12}{*}{3} & 1 & 9 & 2 & 0.3333 & 3 & 1 & 0 & 0 & 0 & 3 & 0 & 1 & 1 & 0 & 3 & & & & & & & $\mathrm{cP}$ & $t P$ \\
\hline & 1 & 8 & 4 & 0.3536 & 4 & 1 & 0 & 0 & 2 & 2 & 0 & 1 & 1 & 0 & 4 & 4 & 0 & 1 & 1 & 0 & 2 & $o C$ & $o C$ \\
\hline & 1 & 5 & 4 & 0.4472 & 5 & 1 & 0 & 0 & 4 & 1 & 0 & 1 & 1 & 0 & 5 & 5 & 0 & 1 & 1 & 0 & 1 & $o C$ & $o C$ \\
\hline & 1 & 4 & 4 & 0.5 & 4 & 1 & 1 & 0 & 2 & 1 & 1 & 1 & 1 & 1 & 4 & 2 & 2 & 1 & 1 & 1 & 2 & $o F$ & $o F$ \\
\hline & 1 & 2 & 4 & 0.7071 & 2 & 1 & 0 & 0 & 2 & 1 & 0 & 1 & 1 & 0 & 2 & 2 & 0 & 1 & 1 & 0 & 1 & $o P$ & $o P$ \\
\hline & 1 & 1 & 8 & 1. & 3 & 1 & 1 & 1 & 3 & 2 & 1 & 1 & 2 & 1 & 1 & & & & & & & $h P$ & $h P$ \\
\hline & 1 & 1 & 4 & 1. & 2 & 1 & 1 & 0 & 2 & 1 & 1 & 2 & 1 & 1 & 2 & 1 & 1 & 1 & 1 & 1 & 1 & $h P$ & $h P$ \\
\hline & 2 & 1 & 4 & 1.4142 & 1 & 1 & 0 & 0 & 2 & 1 & 0 & 2 & 1 & 0 & 1 & 1 & 0 & 1 & 2 & 0 & 1 & $o P$ & $o P$ \\
\hline & 4 & 1 & 4 & 2. & 1 & 1 & 1 & 0 & 2 & 1 & 1 & 4 & 1 & 1 & 1 & 1 & 1 & 2 & 2 & 2 & 1 & $o I$ & $o I$ \\
\hline & 5 & 1 & 4 & 2.2361 & 1 & 1 & 0 & 0 & 4 & 1 & 0 & 5 & 1 & 0 & 1 & 1 & 0 & 1 & 5 & 0 & 1 & $o C$ & $o C$ \\
\hline & 8 & 1 & 4 & 2.8284 & 1 & 2 & 0 & 0 & 2 & 1 & 0 & 4 & 2 & 0 & 1 & 1 & 0 & 2 & 4 & 0 & 1 & $o C$ & $o C$ \\
\hline & 9 & 1 & 2 & 3. & 1 & 3 & 0 & 0 & 0 & 1 & 0 & 3 & 3 & 0 & 1 & & & & & & & $t P$ & $c P$ \\
\hline \multirow[t]{20}{*}{4} & 1 & 16 & 2 & 0.25 & 4 & 1 & 0 & 0 & 0 & 4 & 0 & 1 & 1 & 0 & 4 & & & & & & & $c P$ & $t P$ \\
\hline & 1 & 15 & 4 & 0.2582 & 5 & 1 & 0 & 0 & 2 & 3 & 0 & 1 & 1 & 0 & 5 & 5 & 0 & 1 & 1 & 0 & 3 & $o C$ & $o C$ \\
\hline & 1 & 12 & 4 & 0.2887 & 6 & 1 & 0 & 0 & 4 & 2 & 0 & 1 & 1 & 0 & 6 & 6 & 0 & 1 & 1 & 0 & 2 & $h P$ & $h P$ \\
\hline & 1 & 8 & 2 & 0.3536 & 4 & 1 & 1 & 0 & 0 & 2 & 2 & 1 & 1 & 1 & 4 & & & & & & & $c I$ & $t P$ \\
\hline & 1 & 7 & 4 & 0.3780 & 7 & 1 & 0 & 0 & 6 & 1 & 0 & 1 & 1 & 0 & 7 & 7 & 0 & 1 & 1 & 0 & 1 & $o C$ & $o C$ \\
\hline & 1 & 6 & 4 & 0.4082 & 6 & 1 & 1 & 0 & 4 & 1 & 1 & 1 & 1 & 1 & 6 & 3 & 3 & 1 & 1 & 1 & 2 & $o F$ & $h P$ \\
\hline & 1 & 3 & 8 & 0.5774 & 3 & 1 & 1 & 1 & 1 & 2 & 1 & 1 & 2 & 1 & 3 & & & & & & & $t I$ & $t I$ \\
\hline & 3 & 5 & 4 & 0.7746 & 5 & 3 & 0 & 0 & 2 & 1 & 0 & 1 & 3 & 0 & 5 & 5 & 0 & 3 & 1 & 0 & 1 & $o C$ & $o C$ \\
\hline & 2 & 3 & 4 & 0.8165 & 3 & 1 & 1 & 0 & 4 & 1 & 1 & 2 & 1 & 1 & 3 & 3 & 3 & 2 & 1 & 1 & 1 & $h P$ & $o I$ \\
\hline & 3 & 4 & 4 & 0.8660 & 2 & 1 & 0 & 0 & 4 & 2 & 0 & 3 & 1 & 0 & 2 & 2 & 0 & 1 & 3 & 0 & 2 & $h P$ & $h P$ \\
\hline & 4 & 3 & 4 & 1.1547 & 3 & 2 & 0 & 0 & 4 & 1 & 0 & 2 & 2 & 0 & 3 & 3 & 0 & 2 & 2 & 0 & 1 & $h P$ & $h P$ \\
\hline & 3 & 2 & 4 & 1.2247 & 2 & 1 & 1 & 0 & 4 & 1 & 1 & 3 & 1 & 1 & 2 & 1 & 1 & 1 & 3 & 3 & 2 & $o F$ & $h P$ \\
\hline & 5 & 3 & 4 & 1.2910 & 1 & 1 & 0 & 0 & 2 & 3 & 0 & 5 & 1 & 0 & 1 & 1 & 0 & 1 & 5 & 0 & 3 & $o C$ & $o C$ \\
\hline & 3 & 1 & 8 & 1.7321 & 3 & 3 & 3 & 1 & 1 & 2 & 1 & 3 & 2 & 1 & 1 & & & & & & & $t I$ & $t I$ \\
\hline & 6 & 1 & 4 & 2.4495 & 1 & 1 & 1 & 0 & 4 & 1 & 1 & 6 & 1 & 1 & 1 & 1 & 1 & 2 & 3 & 3 & 1 & $h P$ & $o I$ \\
\hline & 7 & 1 & 4 & 2.6458 & 1 & 1 & 0 & 0 & 6 & 1 & 0 & 7 & 1 & 0 & 1 & 1 & 0 & 1 & 7 & 0 & 1 & $o C$ & $o C$ \\
\hline & 8 & 1 & 2 & 2.8284 & 1 & 2 & 2 & 0 & 0 & 1 & 1 & 4 & 2 & 2 & 1 & & & & & & & $t P$ & $c F$ \\
\hline & 12 & 1 & 4 & 3.4641 & 1 & 2 & 0 & 0 & 4 & 1 & 0 & 6 & 2 & 0 & 1 & 1 & 0 & 2 & 6 & 0 & 1 & $h P$ & $h P$ \\
\hline & 15 & 1 & 4 & 3.8730 & 1 & 3 & 0 & 0 & 2 & 1 & 0 & 5 & 3 & 0 & 1 & 1 & 0 & 3 & 5 & 0 & 1 & $o C$ & $o C$ \\
\hline & 16 & 1 & 2 & 4. & 1 & 4 & 0 & 0 & 0 & 1 & 0 & 4 & 4 & 0 & 1 & & & & & & & $t P$ & $c P$ \\
\hline
\end{tabular}

Consider as an example basal and rhombohedral twins in alumina ceramics (Shiue and Phillips, 1984). $\alpha-\mathrm{Al}_{2} \mathrm{O}_{3}$ has space group $R \overline{3} c$ and axial ratio $c / a=2.730$. The lattice misorientation of the basal twin corresponds to the common rotation with $\Sigma_{h R}=3$. Shiue and Phillips (1984) showed that the Burgers vectors observed in the secondary dislocations of rhombohedral twin boundaries were those predicted by the DSCL of the specific rotation with
$\Sigma_{h R}=7$ that has $\omega=0$ if $(c / a)^{2}=15 / 2$ and $\omega=0.163^{\circ}$ for $c / a=2.730$ (see also Grimmer and Kunze, 2004). Basal and rhombohedral twins have been observed also in hematite iron ore; twins corresponding to the specific $\Sigma_{h P}=7$ rotation that has $\omega=0$ if $(c / a)^{2}=5 / 2$ and $\omega=0.192^{\circ}$ for the axial ratio $c / a=1.587$ of $\mathrm{Ti}$ have been observed in hexagonal metals [see Shiue and Phillips (1984) for references]. 
Table 11. The equivalence classes of symmetry rotations and of common rotations of $h P$ lattices with $\Sigma \leq 30$ and the corresponding classes of $h R$ lattices. The representative rotation of each class has its axis parallel to the principal symmetry axis of the lattice and a minimum rotation angle $\theta$.

\begin{tabular}{lcc}
\hline$\theta\left(^{\circ}\right)$ & $\Sigma_{h P}$ & $\Sigma_{h R}$ \\
\hline 0 & 1 & 1 \\
60 & & 3 \\
21.79 & 7 & 21 \\
38.21 & & 7 \\
27.80 & 13 & 13 \\
32.20 & & 39 \\
13.17 & 19 & 57 \\
46.83 & & 19 \\
\hline
\end{tabular}

\section{Summary}

- The coincidence site lattice (CSL) is a generalization of the twin lattice to misorientations that cannot always be described by a $180^{\circ}$ rotation.

- It deals with geometric aspects of the question: Are there in addition to twin boundaries also other grain boundaries in homophase materials that occur more frequently than at random?

- It helps to understand the dislocation pattern in grain boundaries.

- It is usually considered with materials engineering in mind rather than the interpretation of naturally occurring crystal associations.

\section{Effect of twinning on the diffraction pattern}

The incident beam is diffracted by each individual, and the diffracted waves may be coherent or not depending on the perfection of the individuals and on the wavelength used. Here we concentrate on the case where the waves diffracted by different individuals do not interfere, because otherwise particular problems would result for the structure solution and refinement.

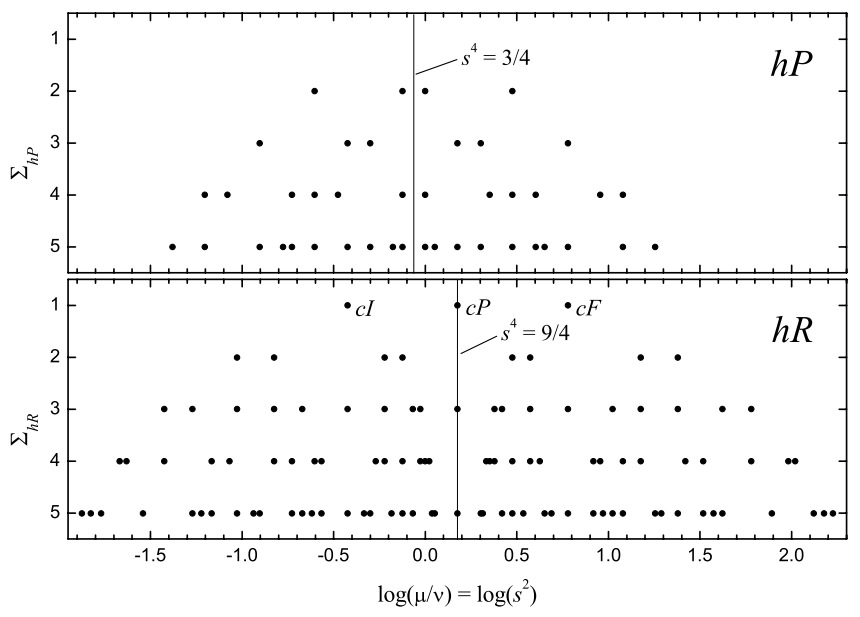

Fig. 9. Specific misorientations of primitive $(h P)$ and rhombohedrally centred $(h R)$ hexagonal lattices with multiplicity (twin index) $\Sigma \leq 5$ as a function of the axial ratio $s$. Notice that the three types of cubic lattices appear as special cases of rhombohedral lattices.
The effect of twinning on the diffraction pattern can ideally be divided into two principal categories:

- TLQS: reflections from different individuals that are related by a twin operation occupy close but not overlapping positions in the diffraction pattern; this effect is known as "splitting" of the reflections and is a strong indication of twinning;

- TLS: reflections from different individuals that are related by a twin operation coincide in the diffraction pattern, i.e. the measured intensity is the sum of the intensities diffracted by the individuals.

The differentiation between these two categories is only ideally neat: for low obliquities, reflections that would be ideally separated can actually be partially overlapping, especially at low 2 angles, and in case of crystals showing deformed reflections departing from the ideal small spherical form (typically, this is the case for samples with structural disorder).

\subsection{Twinning by merohedry}

Let $y$ be a twin operation: its matrix representation will be indicated by the corresponding capital letter $\mathscr{G}$. We indicate by $\langle h k l|$ the row matrix of the coordinates of a node of the reciprocal lattice $\mathbf{L}^{*}$.

Let us start from the simplest case of a two-individual (A and B) twin by merohedry. Each node of $\mathbf{L}^{*}$ is the superposition of a node of $\mathbf{L}^{*}$ A and a node of $\mathbf{L}^{*}{ }_{\mathrm{B}}$. The twin is composed of two individuals: the orientation of the first individual is taken to coincide with the orientation of the twin-lattice, which is fixed in space. Let $\left\langle\left. h k l\right|_{\mathrm{A}}\right.$ be the row matrix of the coordinates of a node $h k l$ of the individual A. The node $\left\langle\left. h k l\right|_{\mathrm{T}}\right.$ of $\mathbf{L}^{*}{ }_{\mathrm{T}}$ corresponds to the superposition of one node from each individual: $\left\langle\left. h k l\right|_{\mathrm{A}}\right.$ from the individual $\mathrm{A}$ and $\left\langle\left. h^{\prime} k^{\prime} l^{\prime}\right|_{\mathrm{B}}\right.$ from the individual $\mathrm{B}$. The reciprocal-lattice coordinates of the nodes of the individual $\mathrm{B}$ with respect to $\mathbf{L}^{*}$ T must be $\langle h k l|$; because the orientation of the individual $\mathrm{B}$ is obtained from that of the individual A by applying $\mathscr{H}$. The node of $\mathrm{B}$ that overlaps $\left\langle\left. h k l\right|_{\mathrm{A}}\right.$ is $\left\langle\left. h^{\prime} k^{\prime} l^{\prime}\right|_{\mathrm{B}}=\left\langle\left. h k l\right|_{\mathrm{A}} \mathscr{G}^{-1}\right.\right.$. The diffraction intensity measured at $\langle h k l|$ is thus the sum of the intensities corresponding to $\left\langle h k l l_{\mathrm{A}}\right.$ and $\left\langle\left. h^{\prime} k^{\prime} l^{\prime}\right|_{\mathrm{B}}\right.$, scaled for the volume fractions, which in case of two individuals are simply $v_{\mathrm{A}}$ and $v_{\mathrm{B}}=1-v_{\mathrm{A}}$ :

$$
I_{h k l}=v_{\mathrm{A}} I_{h k l, \mathrm{~A}}+\left(1-v_{\mathrm{A}}\right) I_{h^{\prime} k^{\prime} l^{\prime}, \mathrm{B}} .
$$

This applies to calculated intensities; measured intensities have to be reduced (absorption, Lorentz, polarization, extinction ...) as usual, in order for the above equation to apply.

Twinning by merohedry is classified, on the basis of the diffractional behaviour, into three classes (Catti and Ferraris, 1976; Nespolo and Ferraris, 2000):

- class I: the twin operation is (equivalent to) an inversion through the origin;

- class IIA: the twin operation is not equivalent to an inversion; it belongs however to $\mathscr{D}$, the holohedry of the (necessarily merohedral) individual;

- class IIB: the individual has a specialized metric and the twin operation belongs to $\mathscr{D}(\mathbf{L})$ but not to $\mathscr{D} \subset \mathscr{D}(\mathbf{L})$.

Twinnings of classes I and IIA correspond to syngonic merohedry, those of class IIB to metric merohedry. The 
measured intensities in case of class I twins are in principle the same as those resulting from an untwinned individual because the diffraction pattern of each individual becomes centrosymmetric according to Friedel's law. It follows that twinning is not at all evident from the diffraction pattern and it may even pass unnoticed in the structural investigation: the structure in fact can be solved and also refined provided that the correct space group has been assumed (Catti and Ferraris, 1976). Resonant scattering has the effect that individuals related by an inversion centre no longer contribute equally to the scattered intensity. This fact is nowadays routinely employed in determining the structures of non-centrosymmetric crystals using synchrotron radiation.

In case of classes IIA and IIB the measured intensities are the sum of non-equivalent reflections and the structure cannot be solved, or at least not refined, without taking the twinning into account. The impossibility of solving or refining the structure is actually a main indication of the possible presence of twinning.

The case of $N$ individuals is the direct generalization of what we described above.

$$
I_{h k l}=\sum_{j=1}^{N} v_{j} I_{h_{j} k_{j} l_{j}, j}, \quad \sum_{j=1}^{N} v_{j}=1 .
$$

\subsection{Twinning by reticular merohedry/ polyholohedry}

When we come to twinning by reticular merohedry/polyholohedry, the indices of the reciprocal lattice nodes have to be expressed in the cell of the reciprocal twin lattice, which is a subcell in reciprocal space. In this cell, the nodes of $\mathbf{L}^{*}$ of a single individual do not take all the possible $h k l$ values, but leave some positions systematically unoccupied. This is simply the result of using a smaller cell for indexing.

Example. Let us take a monoclinic $\mathbf{L}_{\mathrm{I}}$ having $c \cos \beta$ $=-a / 3$. In direct space an orthorhombic triple supercell is obtained by taking an axis $\boldsymbol{c}^{\prime}=\boldsymbol{a}+3 \boldsymbol{c}$ : this supercell defines a possible $\mathbf{L}_{\mathrm{T}}$ (see Fig. 10).

The cell of $\mathbf{L}_{\mathrm{I}}^{*}$ has $a^{*} \cos \beta^{*}=c^{*} / 3$. The subcell defining $\mathbf{L}_{\mathrm{T}}^{*}$ has $\boldsymbol{b}^{* \prime}=\boldsymbol{b}^{*}, \boldsymbol{a}^{* \prime}$ in the $\boldsymbol{a}^{*}-\boldsymbol{c}^{*}$-plane with $\boldsymbol{a}^{* \prime}=$ $a^{*} \sin \beta^{*}$ and $c^{* \prime}=c^{* / 3}$ (see Fig. 11).

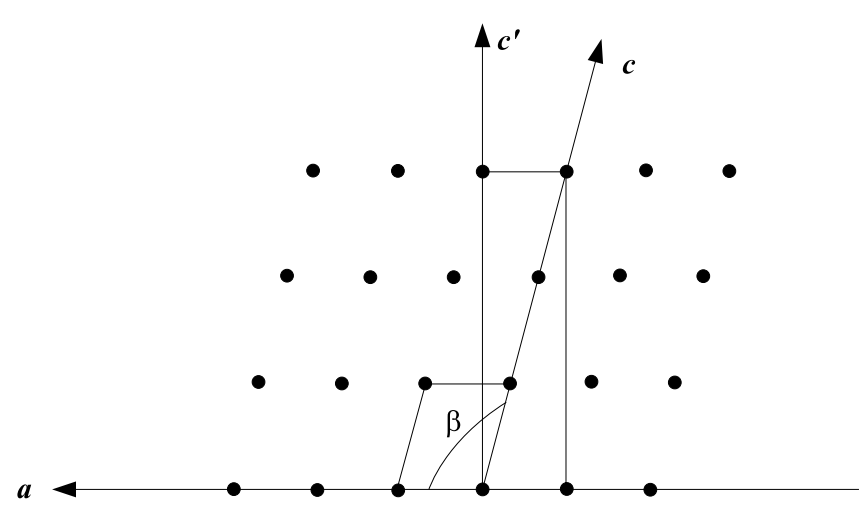

Fig. 10. A monoclinic $P$ cell with $c \cos \beta=-a / 3$, and the corresponding triple orthorhombic supercell projected parallel to the monoclinic axis $\boldsymbol{b}$.

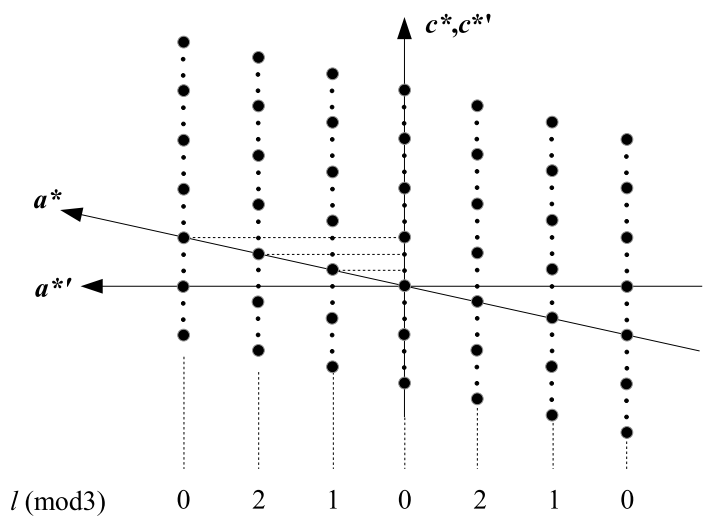

Fig. 11. The reciprocal monoclinic $P$ cell and orthorhombic subcell, corresponding to the cells in Fig. 10, projected parallel to the monoclinic axis $\boldsymbol{b}^{*}$. Large dots correspond to nodes of $\mathbf{L}^{*}$, small dots to additional nodes of $\mathbf{L}^{*}$.

The analysis that follows is made with respect to the cell of $\mathbf{L}^{*}$. The reciprocal lattice nodes of the individual $\left(\mathbf{L}^{*}\right.$ I) occupy one position out of three along $c^{*}$, with $h k l$ satisfying $l=h(\bmod 3)$. Along the rows $1 k l$ (any $k$ ) only positions with $l=3 n+1$ ( $n$ integer) are occupied; along the row $2 k l$ only positions with $l=3 n+2$ ( $n$ integer); and so on.

The derivation of the twin operation is trivial in this case: $\mathscr{D}\left(\mathbf{L}_{\mathrm{I}}\right)=2 / m$ and $\mathscr{D}\left(\mathbf{L}_{\mathrm{T}}\right)=m m m$. The coset decomposition of the latter in terms of the former is:

$$
\begin{aligned}
m m m= & \left\{1,2_{[001]}, 2_{[100]}, 2_{[010]}, \overline{1}, m_{[001]}, m_{[100]}, m_{[010]}\right\} \\
= & \left(2_{[001]}, 2_{[100]}, m_{[001]}, m_{[100]}\right) \cup\left\{1,2_{[010]}, \overline{1}, m_{[010]}\right\} \\
= & 2_{[001]}\left\{1,2_{[010]}, \overline{1}, m_{[010]}\right\} \\
& \cup 2_{[001]}^{2}\left\{1,2_{[010]}, \overline{1}, m_{[010]}\right\},
\end{aligned}
$$

where we have chosen $2_{[001]}$ as representative twin operation among the four equivalent operations defining the twin law. The axes $c^{\prime}$ and $c^{*}$ are parallel and thus the effect of the application of the $2_{[001]}$ twin operation onto $\mathbf{L}^{*}$ is easily visualized. The twin is composed of two individuals: the orientation of the first individual is taken to have its axes $\boldsymbol{b}^{*}$ and $\boldsymbol{c}^{*}$ parallel to those of the space-fixed

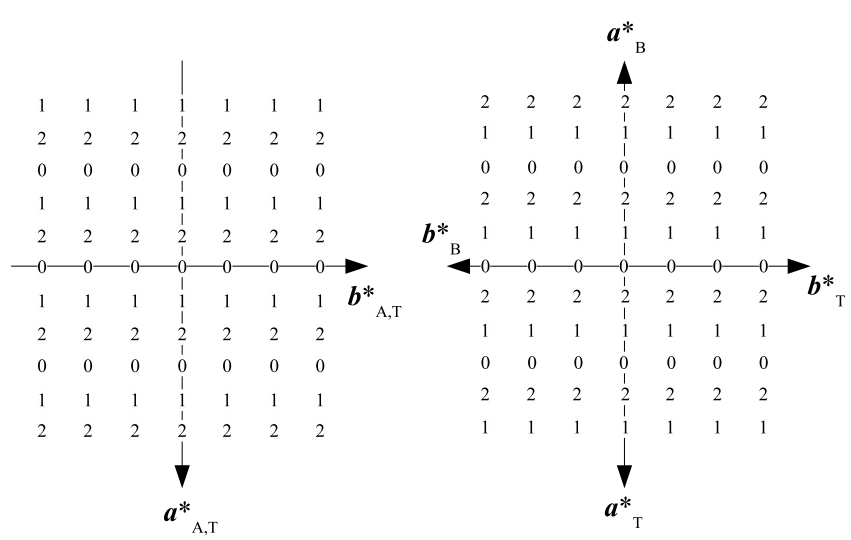

Fig. 12. Projection parallel to $c^{*}$ of the lattice in Fig. 11 in its original orientation and rotated by $180^{\circ}$ about $c^{*}$. "0", "1" and "2" are the $l$ $(\bmod 3)$ indices of the nodes of $\mathbf{L}_{\mathrm{I}}^{*}$ in the axial setting of $\mathbf{L}^{*}$. Notice that $\boldsymbol{a}_{\mathrm{A}}^{*}$ and $\boldsymbol{a}_{\mathrm{B}}^{*}$ are not in the plane $\boldsymbol{a}^{*} \mathrm{~T}^{*}{ }_{\mathrm{T}}$ but inclined by $\beta^{*}$ with respect to $c^{*}$. 
$\mathbf{L}_{\mathrm{T}}: \boldsymbol{b}_{\mathrm{A}}^{*}\left\|\boldsymbol{b}_{\mathrm{T}}^{*}, \boldsymbol{c}_{\mathrm{A}}^{*}\right\| \boldsymbol{c}_{\mathrm{T}}^{*}$. It follows that $\boldsymbol{a}_{\mathrm{A}}^{*}$ lies in the plane $\boldsymbol{a}_{\mathrm{T}}^{*}-\boldsymbol{c}_{\mathrm{T}}^{*}$. The second individual is related to the first one by $\boldsymbol{g}$ and thus $\boldsymbol{b}^{*}{ }_{\mathrm{B}} \|-\boldsymbol{b}^{*}{ }_{\mathrm{T}}$ (Fig. 12).

The twin operation has the effect of dividing $\mathbf{L}_{\mathrm{T}}^{*}$ into three sets (Fig. 13):

1. nodes of $\mathbf{L}^{*}$ T on which nodes from both individuals overlap;

2. nodes of $\mathbf{L}_{\mathrm{T}}^{*}$ that correspond to nodes from only one individual;

3. nodes of $\mathbf{L}^{*}$ that do not correspond to any node of either $\mathbf{L}^{*}{ }_{\mathrm{A}}$ or $\mathbf{L}^{*}{ }_{\mathrm{B}}$.

The third set of nodes gives rise to non-space group absences in the diffraction pattern. Such absences are a strong indication for the presence of twinning.

The coordinates of a node of $\mathbf{L}^{*}{ }_{\mathrm{T}}$ as a function of the nodes of $\mathbf{L}^{*}{ }_{\mathrm{A}}$ and $\mathbf{L}^{*}{ }_{\mathrm{B}}$ are given by the following equation:

$$
\begin{aligned}
& \left\langle\left. h(\bmod 3) k l(\bmod 3)\right|_{\mathrm{T}}\right. \\
& =\left\langle\left. h(\bmod 3) k l(\bmod 3)\right|_{\mathrm{A}}+\left\langle\left. h(\bmod 3) k l(\bmod 3)\right|_{\mathrm{A}} \mathscr{G}^{-1}\right.\right. \\
& =\left\langle\left. h(\bmod 3) k l(\bmod 3)\right|_{\mathrm{A}}+\left\langle\left.(3-h)(\bmod 3) \bar{k} l(\bmod 3)\right|_{\mathrm{B}}\right.\right.
\end{aligned}
$$

Therefore, the lattice nodes of $\mathbf{L}_{\mathrm{T}}^{*}$ receive the contributions given in Table 12 (see Fig. 13):

The result is that along the reciprocal lattice rows parallel to $c^{*}$ having $h \neq 0(\bmod 3)$ one reflection out of three is absent, and along those with $h=0(\bmod 3)$ one out of three is present.

The occurrence of non-space group absences alone is not always a definite criterion for the presence of twinning by reticular merohedry. Let us modify the example above, assuming that $\mathbf{L}_{\mathrm{I}}$ is of type $m C$ with $b=a 3^{1 / 2}$. In this case, the two-dimensional lattice in the (001) plane is of type $h p$ and $\mathbf{L}_{\mathrm{T}}$ is of type $h P$, whence $\mathscr{D}\left(\mathbf{L}_{\mathrm{T}}\right)=6 / \mathrm{mmm}$. We shall use an orthohexagonal cell instead of a primitive hexagonal cell for two reasons: 1) it allows keeping the axes in the (001) plane parallel in $\mathbf{L}_{\mathrm{I}}$ and $\mathbf{L}_{T} ; 2$ ) it makes the following analysis applicable also in the case of TLQS, when the relation $b=a 3^{1 / 2}$ is only approximately obeyed. The coset decomposition becomes:

$$
\begin{aligned}
& \text { 6/mmm } \\
& =6_{[001]}\left\{1,2_{[010]}, \overline{1}, m_{[010]}\right\} \cup 6_{[001]}^{2}\left\{1,2_{[010]}, \overline{1}, m_{[010]}\right\} \\
& \cup 6_{[001]}^{3}\left\{1,2_{[010]}, \overline{1}, m_{[010]}\right\} \cup 6_{[001]}^{4}\left\{1,2_{[010]}, \overline{1}, m_{[010]}\right\} \\
& \cup 6_{[001]}^{5}\left\{1,2_{[010]}, \overline{1}, m_{[010]}\right\} \cup 6_{[001]}^{6}\left\{1,2_{[010]}, \overline{1}, m_{[010]}\right\} \text {. }
\end{aligned}
$$

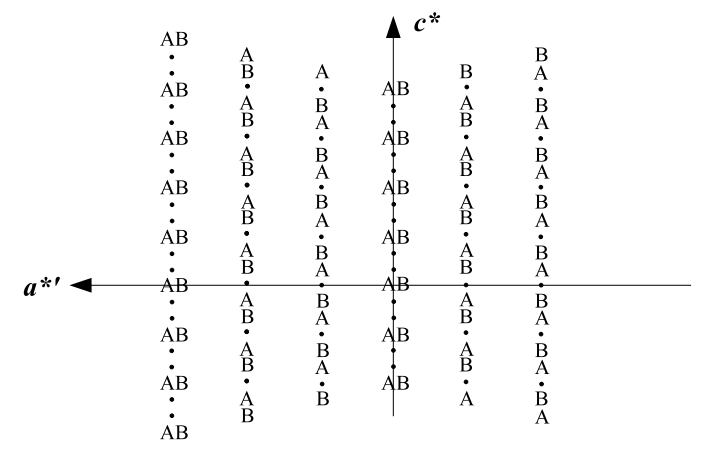

Fig. 13. Overlap of the lattices in Fig. 12.
Table 12. Intensity contributions to the nodes $(h k l)$ of $\mathbf{L}_{\mathrm{T}}^{*}$ coming from two individuals A and B related by $2_{[001]}$.

\begin{tabular}{llll}
\hline$h(\bmod 3)$ & $k$ & $l(\bmod 3)$ & contributions from \\
\hline 1 & any & 0 & none \\
1 & any & 1 & A \\
1 & any & 2 & B \\
2 & any & 0 & none \\
2 & any & 1 & B \\
2 & any & 2 & A \\
0 & any & 0 & A + B \\
0 & any & 1 & none \\
0 & any & 2 & none \\
\hline
\end{tabular}

There are thus five possible twin laws, each corresponding to four equivalent twin operations, which can be expressed by letting five successive powers of $\mathscr{y}=6_{[001]}$ act on $2 / \mathrm{m}$. This is thus a first-degree twin. Let us analyze what happens when we apply these 5 powers of $g$ to $h \mathrm{kl}$. The indi-

\begin{tabular}{|c|c|c|c|}
\hline$h(\bmod 3)$ & $k(\bmod 3)$ & $l(\bmod 3)$ & contributions from \\
\hline 0 & 0 & 0 & $\mathrm{~A}+\mathrm{B}+\mathrm{C}+\mathrm{D}+\mathrm{E}+\mathrm{F}$ \\
\hline 0 & 0 & 1 & none \\
\hline 0 & 0 & 2 & none \\
\hline 1 & 0 & 0 & none \\
\hline 1 & 0 & 1 & $\mathrm{~A}+\mathrm{C}+\mathrm{E}$ \\
\hline 1 & 0 & 2 & $\mathrm{~B}+\mathrm{D}+\mathrm{F}$ \\
\hline 2 & 0 & 0 & none \\
\hline 2 & 0 & 1 & $\mathrm{~B}+\mathrm{D}+\mathrm{F}$ \\
\hline 2 & 0 & 2 & $\mathrm{~A}+\mathrm{C}+\mathrm{E}$ \\
\hline 0 & 1 & 0 & $\mathrm{~A}+\mathrm{D}$ \\
\hline 0 & 1 & 1 & $E+F$ \\
\hline 0 & 1 & 2 & $\mathrm{~B}+\mathrm{C}$ \\
\hline 0 & 2 & 0 & $\mathrm{~A}+\mathrm{D}$ \\
\hline 0 & 2 & 1 & $\mathrm{~B}+\mathrm{C}$ \\
\hline 0 & 2 & 2 & $E+F$ \\
\hline 1 & 1 & 0 & $\mathrm{C}+\mathrm{F}$ \\
\hline 1 & 1 & 1 & $\mathrm{~A}+\mathrm{B}$ \\
\hline 1 & 1 & 2 & $\mathrm{D}+\mathrm{E}$ \\
\hline 1 & 2 & 0 & $\mathrm{~B}+\mathrm{E}$ \\
\hline 1 & 2 & 1 & $\mathrm{~A}+\mathrm{F}$ \\
\hline 1 & 2 & 2 & $C+D$ \\
\hline 2 & 1 & 0 & $\mathrm{~B}+\mathrm{E}$ \\
\hline 2 & 1 & 1 & $C+D$ \\
\hline 2 & 1 & 2 & $\mathrm{~A}+\mathrm{F}$ \\
\hline 2 & 2 & 0 & $\mathrm{C}+\mathrm{F}$ \\
\hline 2 & 2 & 1 & $\mathrm{D}+\mathrm{E}$ \\
\hline 2 & 2 & 2 & $\mathrm{~A}+\mathrm{B}$ \\
\hline
\end{tabular}
viduals $\mathrm{A}, \mathrm{B}, \mathrm{C}, \mathrm{D}, \mathrm{E}, \mathrm{F}$ are each rotated $60^{\circ}$ about $\boldsymbol{c}^{*}$ with respect to the previous one (see Fig. 14).

The presence criteria on $\mathbf{L}_{\mathrm{T}}^{*}$ are as given in Table 13 (see Fig. 15).

Table 13. Intensity contributions to the nodes $(h k l)$ of $\mathbf{L}_{\mathrm{T}}{ }^{*}$ coming from six individuals $\mathrm{A}$ to $\mathrm{F}$ related by $6_{[001]}$. 

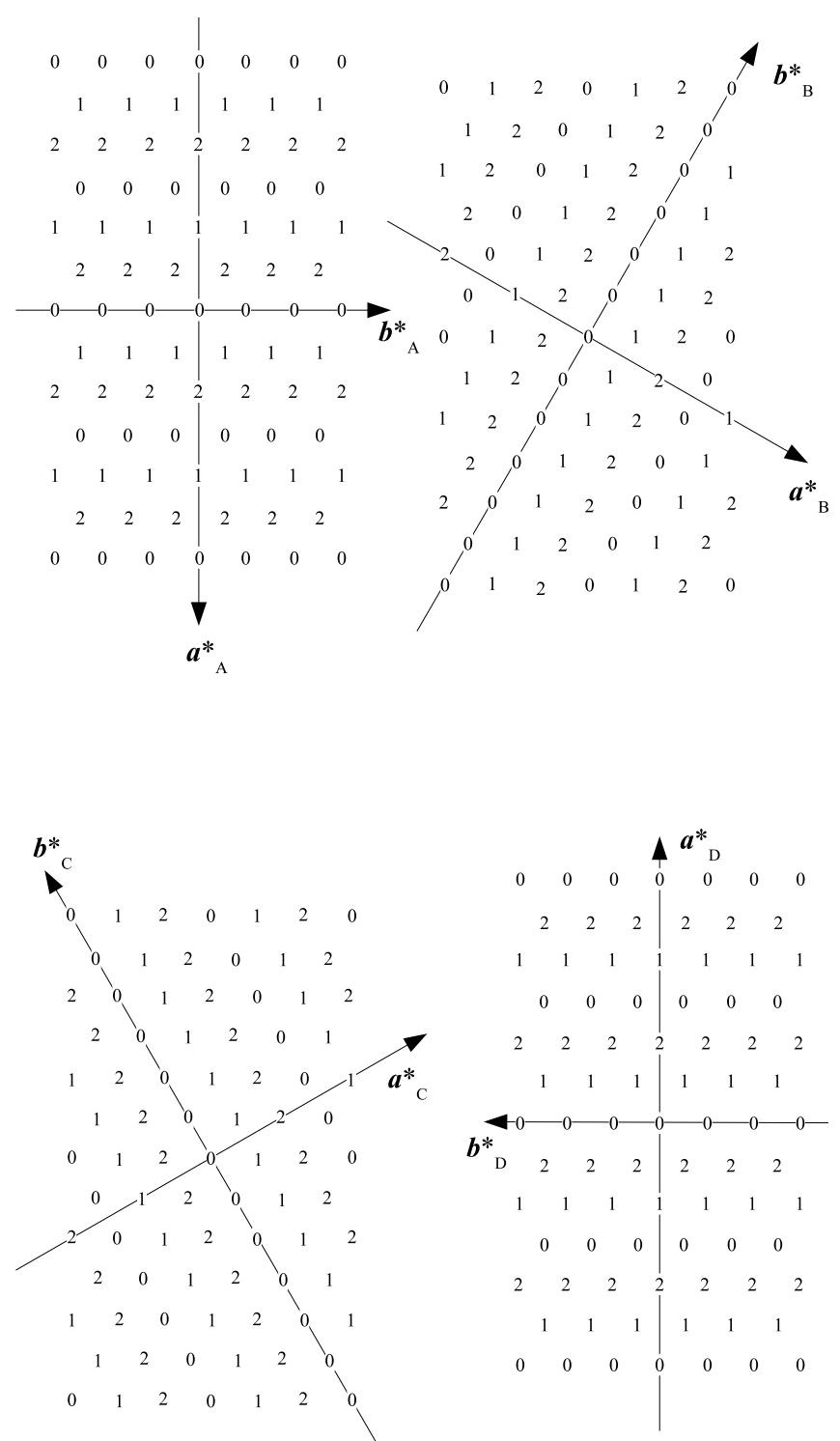

Rows with $k \neq 0(\bmod 3)$ show non-space group absences, suggesting the presence of twinning. However, when only three individuals, at $n \times 120^{\circ}$ from each other (ex. A, C, E), occur, the situation is as given in Table 14 (see Fig. 16).

Now the presence criterion $l=h(\bmod 3)$ if $k=0(\bmod 3)$ is not necessarily related to twinning: it is compatible, for example with local symmetry operations like in OD structures (Dornberger-Schiff, 1979). In a case like this, the geometry of the diffraction pattern alone is not conclusive about the presence of twinning. It suggests instead a possible trigonal symmetry.

Further indications may come from the symmetry of the intensities. For their analysis it is useful to introduce the concept of weighted reciprocal lattice. This is the reciprocal lattice in which each node has a weight corresponding to the resulting intensity (Shmueli, 2001). In particular, a node corresponding to a reflection with zero intensity is omitted from the weighted reciprocal lattice.

Let us analyze the measured intensities in the case of the three individuals described above - with respect to the axes of $\mathbf{L}_{\mathrm{T}}{ }^{*}$ - grouping them according to the trigonal geometry of the diffraction pattern, as shown in Table 15.

Because the symmetry of the individual is only monoclinic, the reflections in each group are not equivalent: $I_{202}$

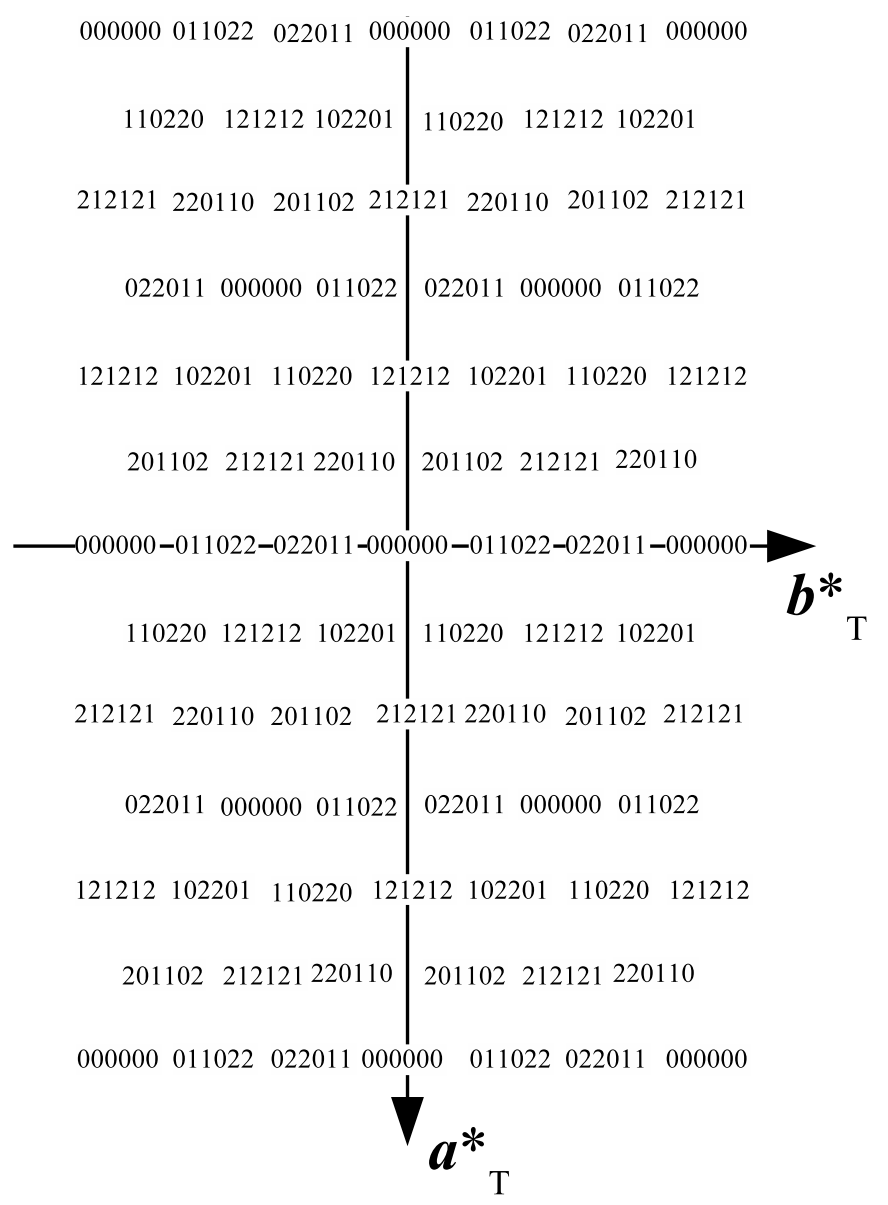

Fig. 15. Overlap of the six lattices in Fig. 14. Each sequence of six numbers corresponds to a row of nodes of $\mathbf{L}^{*}{ }_{\mathrm{T}}$ with the same $h k$ values. The six numbers give the corresponding value of $l(\bmod 3)$ for the 6 individuals.
Fig. 14. Projection parallel to $c^{*}$ of the monoclinic $C$-centred lattice with the metric in the $\boldsymbol{a}-\boldsymbol{c}$-plane as shown in Fig. 10, in six orientations differing by $n \times 60^{\circ}$ rotations about $c^{*}$. Symbols have the same meaning as in Fig. 12. 


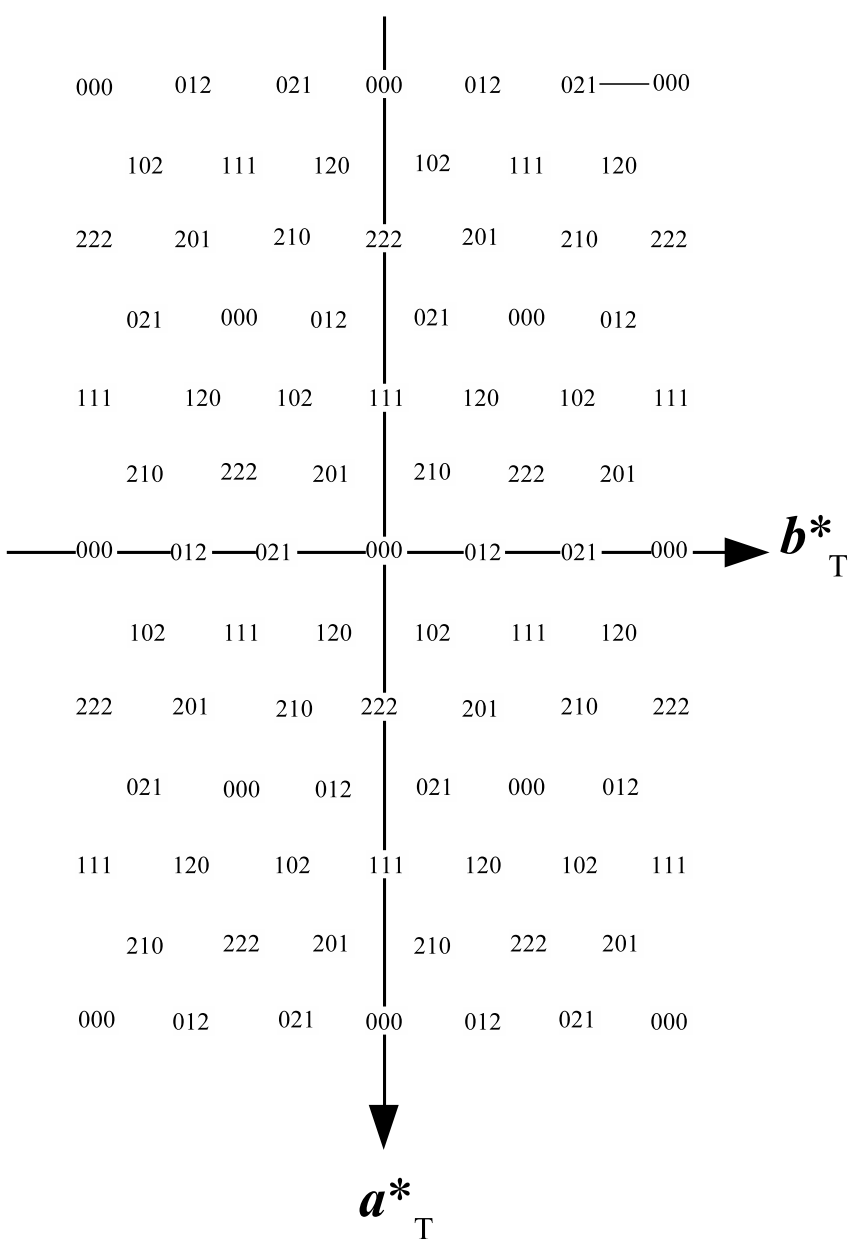

Fig. 16. Overlap of the three lattices on the left side of Fig. 14. Symbols as in Fig. 15.

$\neq I_{1 \overline{3} 2} \neq I_{132} ; I_{131} \neq I_{1 \overline{3} 1} \neq I_{\overline{2} 01}$; etc. Besides, the $h k l$ reflections related by $g$ are scaled by volumes that in general are different: $v_{\mathrm{A}} \neq v_{\mathrm{C}} \neq v_{\mathrm{E}}$. However, in the case of an equi-volume twin, $v_{\mathrm{A}}=v_{\mathrm{C}}=v_{\mathrm{E}}=v=1 / 3$, all three reflections in a group will have the same intensity, simulating thus a trigonal symmetry, not only geometrically, but also by the associated intensities. In a case like this, the presence of twinning is not apparent at all from a preliminary analysis of the diffraction pattern. The structure however cannot be solved, or at least refined, and it is at this stage that the hypothesis of the presence of twinning is formulated.

\section{Conclusions}

Twinning is often considered an obstacle in the daily work of a structural crystallographer, because it causes additional difficulties in the passage from the experimental data to the description and interpretation of the structure. Nevertheless, twinning is itself a challenging phenomenon worth studying, both from the purely crystallographic viewpoint and for its thermodynamic and kinetic aspects influencing crystal growth.

The present review is necessarily far from complete and treats only aspects that are less accessible in a user-
Table 14. Intensity contributions to the nodes $(h k l)$ of $\mathbf{L}_{\mathrm{T}}^{*}$ coming from three individuals $\mathrm{A}, \mathrm{C}$ and $\mathrm{E}$ related by $3_{[001]}$.

\begin{tabular}{|c|c|c|c|}
\hline$h(\bmod 3)$ & $k(\bmod 3)$ & $l(\bmod 3)$ & contributions from \\
\hline 0 & 0 & 0 & $\mathrm{~A}+\mathrm{C}+\mathrm{E}$ \\
\hline 0 & 0 & 1 & none \\
\hline 0 & 0 & 2 & none \\
\hline 1 & 0 & 0 & none \\
\hline 1 & 0 & 1 & $\mathrm{~A}+\mathrm{C}+\mathrm{E}$ \\
\hline 1 & 0 & 2 & none \\
\hline 2 & 0 & 0 & none \\
\hline 2 & 0 & 1 & none \\
\hline 2 & 0 & 2 & $\mathrm{~A}+\mathrm{C}+\mathrm{E}$ \\
\hline 0 & 1 & 0 & A \\
\hline 0 & 1 & 1 & $\mathrm{E}$ \\
\hline 0 & 1 & 2 & $\mathrm{C}$ \\
\hline 0 & 2 & 0 & A \\
\hline 0 & 2 & 1 & $\mathrm{C}$ \\
\hline 0 & 2 & 2 & $\mathrm{E}$ \\
\hline 1 & 1 & 0 & $\mathrm{C}$ \\
\hline 1 & 1 & 1 & $\mathrm{~A}$ \\
\hline 1 & 1 & 2 & $\mathrm{E}$ \\
\hline 1 & 2 & 0 & $\mathrm{E}$ \\
\hline 1 & 2 & 1 & A \\
\hline 1 & 2 & 2 & $\mathrm{C}$ \\
\hline 2 & 1 & 0 & $\mathrm{E}$ \\
\hline 2 & 1 & 1 & $\mathrm{C}$ \\
\hline 2 & 1 & 2 & $\mathrm{~A}$ \\
\hline 2 & 2 & 0 & $\mathrm{C}$ \\
\hline 2 & 2 & 1 & $\mathrm{E}$ \\
\hline 2 & 2 & 2 & $\mathrm{~A}$ \\
\hline
\end{tabular}

Table 15. The intensities diffracted by the individuals $\mathrm{A}, \mathrm{C}$ and $\mathrm{E}$ are grouped according to the trigonal geometry of the diffraction pattern. Notice that $v_{\mathrm{A}}+v_{\mathrm{C}}+v_{\mathrm{E}}=1$.

\begin{tabular}{|c|c|c|}
\hline$I_{000, \mathrm{~T}}=I_{000}$ & $I_{001, \mathrm{~T}}=0$ & $I_{002, \mathrm{~T}}=0$ \\
\hline $\begin{array}{l}I_{110, \mathrm{~T}}=v_{\mathrm{C}} I_{0 \overline{2}} 0 \\
I_{\overline{1} 10, \mathrm{~T}}=v_{\mathrm{E}} I_{0 \overline{2} 0} \\
I_{0 \overline{2} 0, \mathrm{~T}}=v_{\mathrm{A}} I_{0 \overline{2}} 0\end{array}$ & $\begin{array}{l}I_{111, \mathrm{~T}}=v_{\mathrm{A}} I_{111} \\
I_{111, \mathrm{~T}}=v_{\mathrm{C}} I_{111} \\
I_{0 \overline{2} 1, \mathrm{~T}}=v_{\mathrm{E}} I_{111}\end{array}$ & $\begin{array}{l}I_{112, \mathrm{~T}}=v_{\mathrm{E}} I_{\overline{1} 12} \\
I_{112, \mathrm{~T}}=v_{\mathrm{A}} I_{\overline{1} 12} \\
I_{0 \overline{2} 2, \mathrm{~T}}=v_{\mathrm{C}} I_{\overline{1} 12}\end{array}$ \\
\hline $\begin{array}{l}I_{020, \mathrm{~T}}=v_{\mathrm{A}} I_{020} \\
I_{\overline{1} \overline{1} 0, \mathrm{~T}}=v_{\mathrm{C}} I_{020} \\
I_{1 \overline{1} 0, \mathrm{~T}}=v_{\mathrm{E}} I_{020}\end{array}$ & $\begin{array}{l}I_{021, \mathrm{~T}}=v_{\mathrm{C}} I_{1 \overline{1} 1} \\
I_{\overline{1} 1 \overline{1} 1, \mathrm{~T}}=v_{\mathrm{E}} I_{1 \overline{1} 1} \\
I_{1 \overline{1} 1, \mathrm{~T}}=v_{\mathrm{A}} I_{1 \overline{1} 1}\end{array}$ & $\begin{array}{l}I_{022, \mathrm{~T}}=v_{\mathrm{E}} I_{\overline{1} 1} 2 \\
I_{\overline{1} \overline{1} 2, \mathrm{~T}}=v_{\mathrm{A}} I_{\overline{1} 1} 2 \\
I_{1 \overline{1} 2, \mathrm{~T}}=v_{\mathrm{C}} I_{\overline{1} 1} \overline{1} 2\end{array}$ \\
\hline $\begin{array}{l}I_{310, \mathrm{~T}}=v_{\mathrm{A}} I_{310} \\
I_{240, \mathrm{~T}}=v_{\mathrm{C}} I_{310} \\
I_{150, \mathrm{~T}}=v_{\mathrm{E}} I_{310}\end{array}$ & $\begin{array}{l}I_{311, \mathrm{~T}}=v_{\mathrm{E}} I_{\overline{2} 41} \\
I_{241, \mathrm{~T}}=v_{\mathrm{A}} I_{241} \\
I_{151, \mathrm{~T}}=v_{\mathrm{C}} I_{241}\end{array}$ & $\begin{array}{l}I_{312, \mathrm{~T}}=v_{\mathrm{C}} I_{1 \overline{5} 2} \\
I_{242, \mathrm{~T}}=v_{\mathrm{E}} I_{\overline{1} 52} \\
I_{1 \overline{1} 2, \mathrm{~T}}=v_{\mathrm{A}} I_{1 \overline{1}}\end{array}$ \\
\hline $\begin{array}{l}I_{3 \overline{1} 0, \mathrm{~T}}=v_{\mathrm{A}} I_{3 \overline{1} 0} \\
I_{150, \mathrm{~T}}=v_{\mathrm{C}} I_{3 \overline{1} 0} \\
I_{2 \overline{4} 0, \mathrm{~T}}=v_{\mathrm{E}} I_{3 \overline{1} 0}\end{array}$ & $\begin{array}{l}I_{3 \overline{1} 1, \mathrm{~T}}=v_{\mathrm{C}} I_{2 \overline{4} 1} \\
I_{151, \mathrm{~T}}=v_{\mathrm{E}} I_{\overline{2}} \overline{2}_{1} 1 \\
I_{2 \overline{4} 1, \mathrm{~T}}=v_{\mathrm{A}} I_{2 \overline{4} 1}\end{array}$ & $\begin{array}{l}I_{3 \overline{1} 2, \mathrm{~T}}=v_{\mathrm{E}} I_{152} \\
I_{152, \mathrm{~T}}=v_{\mathrm{A}} I_{\overline{1} 52} \\
I_{\overline{2} \overline{4} 2, \mathrm{~T}}=v_{\mathrm{C}} I_{\overline{1} 52}\end{array}$ \\
\hline
\end{tabular}

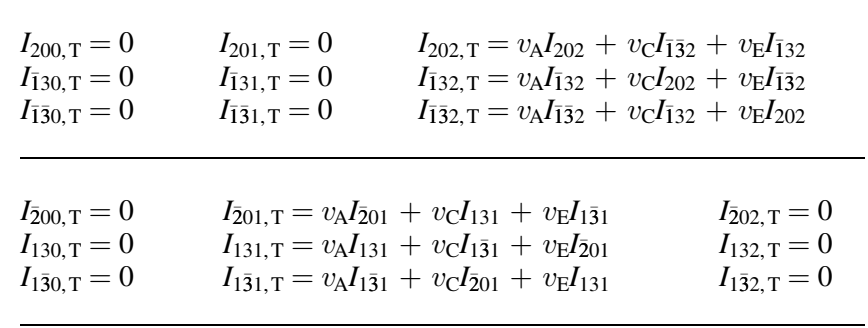


friendly form elsewhere. We nevertheless hope to have shown the advantage of a stronger geminographical background, not only for the theoretical crystallographer but also for the structural investigator.

Acknowledgments. The authors are grateful to the referees for their constructive criticism, from which the present text has profited considerably.

\section{References}

Bollmann, W.: On the geometry of grain and phase boundaries I. General theory. Philos. Mag. 16 (1967a) 363-381.

Bollmann, W.: On the geometry of grain and phase boundaries II. Applications of general theory. Philos. Mag. 16 (1967b) 383399.

Bollmann, W.: Crystal Defects and Crystalline Interfaces. SpringerVerlag, Berlin 1970.

Brandon, D. G.: The structure of high-angle grain boundaries. Acta Metall. 14 (1966) 1479-1484.

Brandon, D. G.; Ralph, B.; Ranganathan, S.; Wald, M. S.: A field ion microscope study of atomic configuration at grain boundaries. Acta Metall. 12 (1964) 813-821.

Bravais, M. A.: Mémoire sur les systèmes formés par des points distribués régulièrement sur un plan ou dans l'espace. J. École Polytechn. 19, Cahier 33 (1850) 1-128 (English translation: On the systems formed by points regularly distributed on a plane or in space, translated by Amos J. Shaler, Crystallographic Society of America, Memoir Number One, The Book Concern, Hancock, Michigan 1949).

Buerger, M. J.: The diffraction symmetry of twins. Anais acad. brasil. cienc. 26 (1954) 111-121 [republished in Buerger, M. J.: Crystalstructure analysis, John Wiley \& Sons, New York (1960) 53-67].

Buerger, M. J.: Image sets. Z. Kristallogr. 116 (1961) 430-467.

Catti, M.; Ferraris, G.: Twinning by merohedry and X-ray crystal structure determination. Acta Crystallogr. A32 (1976) 163-165.

Coxeter, H. S. M.: Regular Polytopes, $3^{\text {rd }}$ edition, Dover Publication Inc., New York 1973.

Coxeter, H. S. M.: Introduction to Geometry, $2^{\text {nd }}$ edition, John Wiley and Sons, Inc., New York 1989.

Curien, H.; Le Corre, Y.: Notations des macles à l'aide du symbolisme des groupes de couleurs de Choubnikov. Bull. Soc. Franç. Minér. Crist. 81 (1958) 126-132.

Donnay, J. D. H.: Width of Albite-Twinning Lamellae. Am. Mineral. 25 (1940) 578-586.

Donnay, J. D. H.; Donnay, G.: Twinning, In: International Tables for $X$-Ray Crystallography, Vol. III. pp. 104-105. Kynoch Press, Birmingham 1959.

Donnay, G.; Donnay, J. D. H.: Classification of triperiodic twins. Can. Mineral. 12 (1974) 422-425.

Dornberger-Schiff, K.: OD structures - a game and a bit more. Krist. Tech. 14 (1979) 1027-1045.

Fischmeister, H. F.: Structure and properties of high angle grain boundaries. J. Phys., Paris 46, C4 (1985) C4-3-C4-23.

Flack, H. D.: The derivation of twin laws for (pseudo-)merohedry by coset decomposition. Acta Crystallogr. A43 (1987) 564-568.

Fortes, M. A.: Coincidence site lattices in non-cubic lattices. phys. stat. sol. (b) 82 (1977) 377-382.

Fortes, M. A.: Crystallographic applications of the elementary divisor theorem. Acta Crystallogr. A39 (1983a) 348-350.

Fortes, M. A: N-dimensional coincidence-site-lattice theory. Acta Crystallogr. A39 (1983b) 351-357.

Friedel, G.: Étude sur les groupements cristallins. Extrait du Bulletin de la Société de l'Industrie minérale, Quatrième série, Tomes III e IV, Societé de l'Imprimerie Théolier - J. Thomas et $C^{\text {ie }}$, SaintÉtienne 1904.

Friedel, G.: Sur les macles du quartz. Bull. Soc. Franç. Minér. 46 (1923) 79-95.

Friedel, G.: Leçons de Cristallographie, Berger-Levrault, Nancy, Paris, Strasbourg 1926.
Frondel, C.: The System of Mineralogy of J. D. and E. S. Dana, v. 3, Silica Minerals: $7^{\text {th }}$ edition, John Wiley and Sons, New York 1962.

Giacovazzo, C.: Fundamentals of Crystallography. $2^{\text {nd }}$ edition, Oxford Science Publications, Oxford 2002.

Grimmer, H.: Coincidence rotations for cubic lattices. Scripta Metall. 7 (1973) 1295-1300.

Grimmer, H.: A reciprocity relation between the coincidence site lattice and the DSC lattice. Scripta Metall. 8 (1974) 1221-1223.

Grimmer, H.: Coincidence-Site Lattices. Acta Crystallogr. A32 (1976) $783-785$.

Grimmer, H.: A unique description of the relative orientation of neighbouring grains. Acta Crystallogr. A36 (1980) 382-389.

Grimmer, H.: Remarks on coincidence orientations of tetragonal and orthorhombic lattices. Materials Science Forum 126-128 (1993) 269-272.

Grimmer, H.: Determination of all misorientations of tetragonal lattices with low multiplicity; connection with Mallard's rule of twinning. Acta Crystallogr. A59 (2003) 287-296.

Grimmer, H.; Kunze, K.: Twinning by reticular pseudo-merohedry in trigonal, tetragonal and hexagonal crystals. Acta Crystallogr. A60 (2004) 220-232.

Hahn, T.: International Tables for Crystallography, Vol. A, $5^{\text {th }}$ edition. Kluwer Academic Publishers, Dordrecht 2002.

Hahn, T.; Klapper, H.: Twinning of crystals. In: International Tables for Crystallography, Vol. D. (Ed. A. Authier), pp. 393-448. Kluwer Academic Publishers, Dordrecht 2003.

Koch, E.: Twinning. In: International Tables for Crystallography, Vol. C, $3^{\text {rd }}$ edition (Ed. E. Prince), pp. 10-14. Kluwer Academic Publishers, Dordrecht 2004.

Kronberg M. L.; Wilson, F. H.: Secondary recrystallization in copper. Trans. A.I.M.E. 185 (1949) 501-514.

Mügge, O.: Über Translationen und verwandte Erscheinungen in Krystallen. Neues Jahrb. Mineral. Geol. Paläontol. 1 (1898) 71-158.

Mykura, H.; Bansal, P. S.; Lewis, M. H.: Coincidence-site-lattice relations for $\mathrm{MgO}-\mathrm{CdO}$ interfaces. Philos. Mag. A42 (1980) 225-233.

Nespolo, M.: Twin point groups and the polychromatic symmetry of twins. Z. Kristallogr. 219 (2004) 57-71.

Nespolo, M.; Ferraris, G.: Twinning by syngonic and metric merohedry. Analysis, classification and effects on the diffraction pattern. Z. Kristallogr. 215 (2000) 77-81.

Nespolo, M.; Ferraris, G.: Geminography - The science of twinning applied to the early-stage derivation of non-merohedric twin laws. Z. Kristallogr. 218 (2003) 178-181.

Nespolo, M.; Ferraris, G.: Applied geminography - Symmetry analysis of twinned crystals and definition of twinning by reticular polyholohedry. Acta Crystallogr. A60 (2004) 89-95.

Nespolo, M.; Ferraris, G.; Takeda, H.; Takéuchi, Y.: Plesiotwinning: oriented crystal associations based on a large coincidence-site lattice. Z. Kristallogr. 214 (1999) 378-382.

Pedersen, B. F.; Semmingsen, D.: Neutron diffraction refinement of the structure of gypsum, $\mathrm{CaSO}_{4} \cdot 2 \mathrm{H}_{2} \mathrm{O}$. Acta Crystallogr. B38 (1982) 1074-1077.

Pies, W.; Weiss, A.: Crystal structure data of inorganic compounds. In: Landolt-Börnstein, New Series, Vol. III/7d1 $\alpha$, pp. 120-122. Springer-Verlag, Berlin 1985.

Reed, B. W.; Minich, R. W.; Rudd, R. E.; Kumar, M.: The structure of the cubic coincident site lattice rotation group. Acta Crystallogr. A60 (2004) 263-277.

Shiue, Y. R.; Phillips, D. S.: The crystallography of annealing twins in alumina ceramics. Philos. Mag. A50 (1984) 225-233.

Shmueli, U.: International Tables for Crystallography, Vol. B, $2^{\text {nd }}$ edition. Kluwer Academic Publishers, Dordrecht 2001.

Singh, A.; King, A.: Tables of coincidence orientations for ordered tetragonal $\mathrm{L} 1_{0}$ alloys for a range of axial ratios. Acta Crystallogr. B49 (1993) 266-272.

Sokolova, E.; Hawthorne, F. C.: The crystal chemistry of epistolite. Can. Mineral. 42 (2004) 797-806.

Takeda, H.: Prediction of twin formation. J. Mineral. Soc. Japan 12 (1975) 89-102 (in Japanese).

Takeda, H.; Donnay, J. D. H.: Compound tessellations in crystal structures. Acta Crystallogr. 19 (1965) 474-476. 